\title{
LATE BREAKING ABSTRACTS
}

\section{Information Technology - LBA}

\section{LBP-1 The Construction and Development of Information System of Clinical Biobank}

D. Guo, Y. Xu, Z. Fan, Z. Liang, S. Zhang

\section{PUMCH, Beijing, China}

Background: Due to the rapid development of translational medicine research, the construction and application clinical biobanks have increasing attention. The information system is the core of the biobank, which plays an important role on sharing of clinical information and specimens. So the development of clinical biobank information system should based on supporting the overall process of operation, management and service.

Methods: The multifunctional requirements of the clinical biobank need powerful information system to track the full life cycle of biospecimen from collection, reception, transportation, processing, storage and distribution. So, the function of information system should include management of biospecimen, clinical data, molecular data, materials, personnel, equipment finance, and so on. Biospecimen management system should take sample as center, and Clinical data management take patients as center. Besides common data element standardization is the basic point of information system, which also has characters of flexibility, openness and safety.

To construct the information system, the sample/data collection flow should be considered firstly. When the patient enter the hospital through the ADT (Admission, Discharge, Transfer System), doctors recruit them to different research projects according to clinical phenotype and diagnosis, sign informed consent, and begin the sample collecting procedure with CPOE (Computerized Physician Order Entry). With the acquiring of clinical data and diagnosis from AIMS(Anesthesia Information Management System), EMR(Electronic Medical Record), PIS (Pathology Information System), clinical biobank could access the quality of the biospecimen, then judge whether would be archive.

Results: Compared with the traditional biobank which rely on manual input data, new generation biobank are more focused on the quality and integrity of data. The information system joining the sample and data collection process with ADT, CPOE, EMR, LIMS, AIMS, PIS, PACS and MRMS could not only acquire automatically, but also guarantee the reliability, timeliness and accuracy of the data.

Conclusion: A powerful biobank information system could realize the efficient management and promote comprehensive sharing of biospecimen, clinical information and molecular data, which would take advantage of the era of big data to support biomarkers detection, individualized medical treatment, drug research and development.

\section{LBP-2 Comprehensive Paperless Biorepository Management}

\author{
C. Thomas, S. Thomas, J. Lamptey, R. Indibi, L. Colligan, \\ W. Shao
}

Preclinical Development, Regeneron Pharmaceuticals, Inc, Tarrytown, New York, United States

Background: Regeneron's Sample Management Group (SMG) manages sample receipts, requests, and transfers, and maintains short- and long-term storage for samples from both non-clinical studies and clinical trials. The increase in number of samples that are received daily from central Labs and the requests for sample transfers within multiple departments at Regeneron along with the rising complexity of sample tracking from acquisition through destruction have necessitated process changes and/or improvements to increase efficiency and minimize errors while maintaining compliance.

Methods: Paper based sample management process is no longer sustainable for handling an ever increasing number of samples. In order to maximize efficiency and ensure that data is captured accurately in a Laboratory Information Management System (LIMS), a paperless sample receipt and sample request process was developed and implemented. The process includes the use of electronic forms in LIMS, which facilitate tracking accuracy from the time samples arrive through disposal.

Results: Implementation of paperless sample receipt and request forms has allowed SMG to document and track shipments, and pull requested samples more efficiently and with fewer errors. The paperless sample receipt form prompts the user for electronic data entry of all shipment information including quantity of samples received and short-term storage location. The paperless sample request form matches each scanned barcode to the barcodes listed in the request, and ensures that the samples are in the correct order and position. The paperless sample request also facilitates the timely and automatic communication of the job status to the requestors and SMG team.

Conclusion: The use of paperless sample receipt and request forms in LIMS has dramatically improved efficiency and accuracy. Through this significant process improvement sample management professionals and biobank communities have been able to streamline processes to maintain high throughput, increase sample visibility, and compliance despite increasing sample quantities.

\section{LBP-3 A Comprehensive Biobanking Ontology of the Lifecycle of the Biospecimen Made for-and-with Diverse Subject Matter Experts}

H. J. Ellis ${ }^{1}$, M. Brochhausen ${ }^{5}$, A. Masci ${ }^{3}$, F. J. Manion ${ }^{6}$, J. Obeid ${ }^{4}$, C. J. Stoeckert Jr. ${ }^{2}$, J. Zheng ${ }^{2}$

${ }^{1}$ Biobanking Without Borders, LLC, Durham, North Carolina, United States, ${ }^{2}$ University of Pennsylvania, Philadelphia, Pennsylvania, United States, ${ }^{3}$ Department of Biostatistics 
and Bioinformatics, Duke University, Durham, North Carolina, United States, ${ }^{4}$ Medical School of South Carolina, Charleston, South Carolina, United States, ${ }^{5}$ University of Arkansas for Medical Sciences, Little Rock, Arkansas, United States, ${ }^{6}$ University of Michigan Comprehensive Cancer Center, Ann Arbor, Michigan, United States

Problem Statement: The field of biobanking demands data integration. Biobanks commonly evolve for diverse research needs with heterogeneous data representations presenting challenges to query for samples and associated clinical annotations across biobanks, within and across institutions. Moreover, there are ethical and regulatory requirements for the use of specimens and data, including documentation and adherence to the permissions and restrictions expressed by research participants during consent.

Proposed Solution: The Ontology for BioBanking (OBIB) has been developed and refined through engagement and collaboration with several academic medical institutions, national scientific biobanking forums and national standards organizations. An ontology provides clearly defined terms and the relationships between them in a computer-interpretable way, enabling integration of disparate data into one harmonized, reusable system. OBIB was developed following principles of the Open Biomedical Ontologies Foundry, which requires reuse of existing interoperable ontologies in order to prevent multiple representations of the same entities. The scope of OBIB is the Lifecycle of the Biospecimen as defined by NCI's Biorepositories and Biospecimen Research Branch (BBRB), starting with the participant consent process. Important aspects of informed consent are addressed by cross-linking with the Informed Consent Ontology (ICO). OBIB leverages work done on data elements from biobanks at academic institutions as well as the NIH Genotype-Tissue Expression (GTEx) Project and the NCI's BBRB. The contributions from GTEx include comprehensive research authorizations related to postmortem tissue donation, and terms from the BBRB relate to pathology staging and grading, specimen annotation and medical history. OBIB classes are being linked to terms in MIABIS (Minimum Information About BIobank data Sharing) version 2.0. OBIB currently contains 1970 terms including 1617 classes, of which 130 terms originated from Ontologized MIABIS (OMIABIS) and 1234 were imported from other ontologies.

Conclusion: The continued development of OBIB benefits from the diverse members of the collaboration. OBIB provides a solution for identifying specimens for research, and effectively using the wealth of information present in biobanks by integrating data stored in those repositories, with clinical data and relevant consents. OBIB is freely available at https:// bioportal.bioontology.org/ontologies/OBIB.

\section{LBP-4 Transforming Sample Creation Process for Biobanking Registration with Excel Macros}

Y. Liu, E. Shum, R. DeMarte, M. Ngo, S. Valluzzo, L. Colligan, W. Shao

Preclinical Development, Regeneron, Tarrytown, New York, United States

Statement of the Problem: When Nautilus Laboratory Information Management System (LIMS) is used to manage study samples received from different central Labs or contract research organizations (CROs), creation of new samples in preparation for pre-registration of thousands of samples in LIMS has typically been a manual process. This is because the different systems used by CROs, which generate sample listing files in different formats, make it very challenging to automate the sample creation process. LIMS samples can be created by importing sample information in Microsoft Excel with a format required by LIMS configuration. However, the manual creation of Excel files can be time consuming and introduce human error for shipments with large quantities of samples. The repetitive operations in Excel also pose high ergonomic risk.

Proposed Solution: Macros written with Excel VBA (Visual Basic for Applications) were used to automate steps during Excel file creation in order to increase efficiency and accuracy. The macros extract required sample information from multiple electronic shipping manifests and combine all the information into a single Excel worksheet. The macros then format sample information, e.g. site, subject, visit, time point, sample collection date, etc., and generate a file that can be imported into LIMS to create new samples and vials. The automated process can reduce hours of manual work into seconds without an error, depending on the size of the files, the number of samples and the number of shipments. It also replaces repetitive manual operations in Excel to lower ergonomic risk.

The macros also enable flags that require users' attention and provide the flexibility of manual updates if there are errors or discrepancies in the manifests. The macros were designed with user friendly interface that simplifies the training process.

Conclusions: Manual creation of new samples in LIMS can be very time consuming and is prone to human errors. Excel Macros are very efficient tools to automate the steps to create Excel files with new sample information that can be imported in LIMS. The macros with simplified interface are very helpful to harmonize the file creation process, increase efficiency, minimize human errors and lower users' ergonomic risks.

\section{LBP-5 OpenSpecimen - Experiences of Collaborative Development of an Open Source Biobanking Informatics Platform}

\section{S. Adiga}

\section{Krishagni Solutions Pvt Ltd, Pune, Maharashtra, India}

Access to high-quality biospecimens with associated data annotations is crucial for research. Recent advances in molecular biology and genetics have resulted in a concomitant increase in the demand for well-annotated, properly preserved specimens. Today biobanking is a highly dynamic activity which faces many challenges, including the need to deal with ever increasingly complex demands of managing data and integrations with existing databases.

The available informatics solutions will not have an "out of the box" support or sufficient data elements set up appropriately. The informatics platform will need to support the complex sample management workflows and data collection needs which are of diverse nature and specific to each collaborator, disease, or even geographic location.

OpenSpecimen is the result of the collaborative efforts of NCI and has continued its further evolution with industry and academic partnership. For the past eight years, Krishagni has worked closely with its biobanking community to develop a robust, scalable and highly flexible open source biobanking informatics platform.

As a result, OpenSpecimen is today used in $65+$ biobanks across 15 countries.

Open source software (OSS) promotes collaboration, avoids single "vendor lock-in" and drives the cost of ownership down. It ensures a higher level of security since the source code is 
publicly available for audit. In comparison, proprietary software is highly secretive, can only be customized or enhanced by the vendor, usually at a prohibitive cost. In many instances, adopters are left with no option when the vendor ceases operation or decides to focus on some other product or business.

In this poster, we will demonstrate how collaboration with biobanks across the globe has allowed OpenSpecimen to expand and meet the ever-increasing needs of this domain. We will present examples of collaboration with Johns Hopkins, Memorial Sloan Kettering, Children's Hospital (Dallas), UT Southwestern (USA), University of New South Wales, SAHMRI (Australia), Singapore General Health and University of Leicester (United Kingdom). The poster will also highlight the open source methodology and the enhancements developed in OpenSpecimen as part of these collaborations.

In summary, this poster will highlight an increased need for informatics systems to stay apace with the changes being experienced by biobanking societies and how OpenSpecimen uses open source to achieve collaboration amongst biobanks across the globe.

\section{LBP-6 Data Quality Assessment of the Human Biobank Information System in the National Biobank of Korea}

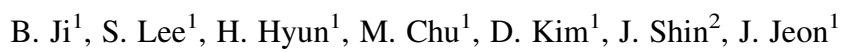

${ }^{1}$ Korea National Institute of Health, Cheongju-si, Chungcheongbuk-do, Korea, ${ }^{2}$ Ministry of Health \& Welfare, Sejong-si, Korea

Most biobanks are manipulating biospecimen-related inventory data by manual into the informatics system, which may generate various errors in the database.

The National Biobank of Korea (NBK) has operated the selfdeveloped Human Biobank Informatics System (HuBIS) which maintains the storage and dissemination information of biological samples. This HuBIS handles various biobank inventory data generated from a central biobank and 17 regional biobanks which comprised of the Korea Biobank Network (KBN). Here, we report the analysis results of the data quality and database structure of the HuBIS in order to improve the database quality and reliability. The HuBIS database was analyzed for pattern of data errors in terms of 12 assessment areas including uniqueness and column consistency according to the Database Quality Certification-Value (DQC-V) of the Korea Data Agency. As the assessment result, the uniqueness was $0.17 \%$ and column consistency was $3.3 \%$. The error rate of the total evaluation standard was $3.04 \%$, which is equivalent to 3.2 of a Sigma level and the Silver class of the DQC-V standard. In addition, we analyzed the Entity Relationship Diagram (ERD) of the database structure, showing that it is possible to increase the data quality efficiently by improving data normalization.

Based on the assessment results of database quality, we will apply for a data quality certification of the Korea Data Agency, and will implement the 5-year roadmap of data quality management of the HuBIS.

\section{LBP-7 Reducing the Human Data Entry Burden of a Growing Pediatric Brain Tumor Biospecimen Banking Consortium}

A. S. Felmeister ${ }^{1,2}$, S. Leary ${ }^{3,4}$, J. Stevens ${ }^{4}$, J. Mason ${ }^{1}$, R. E. Teneralli ${ }^{1}$, C. Bailey ${ }^{1,5}$, A. J. Waanders ${ }^{1,5}$
${ }^{1}$ The Children's Hospital of Philadelphia, Philadelphia, Pennsylvania, United States, ${ }^{2}$ College of Computing and Informatics, Drexel University, Philadelphia, Pennsylvania, United States, ${ }^{3}$ Fred Hutchinson Cancer Research Center, University of Washington School of Medicine, Seattle, Washington, United States, ${ }^{4}$ Seattle Children's Hospital, Seattle, Washington, United States, ${ }^{5}$ Department of Pediatrics, University of Pennsylvania Perelman School of Medicine, Philadelphia, Pennsylvania, United States

Background: Researchers at Children's Hospital of Philadelphia (CHOP) and Seattle Children's Hospital (SCH) are collaborating on a project to inform diagnostics and treatment options of pediatric supratentorial malignant cortical brain by correlating molecular results and longitudinal outcome data. The source of the tissue is the Children's Brain Tumor Tissue Consortium (CBTTC). Data from the CBTTC is manually abstracted prospectively from surgery through treatment. In this study we aim to automate the annotation of biospecimens by leveraging observations in Clinical Data Research Networks (CDRNs).

Methods: We are focusing the effort of this research on the CDRN, PEDSnet, where two CBTTC specimen collection sites participate. We obtained simulated patient data in the model used by PEDSnet. We performed an exploratory data analysis of this data ran multiple algorithms to predict a specific condition in a patient based on frequencies and sequences of events. Thus creating pipelines to distinguish data-driven phenotypes of specific subsets of patients. Concurrently, a distinct list of histological conditions was mapped from the CBTTC terminology to SNOMED-CT that was preliminarily hand-validated with neuro-oncologists from both $\mathrm{CHOP}$ and $\mathrm{SCH}$ for an initial request of data from PEDSnet.

Results: The observational data model lent itself to straightforward visualizations at the individual and population levels, and patient-based analyses for disease and comparison with te rest of the population. These data points successfully fit Kaplan-Meier curves for any disease qualification category. Timelines were also created at the individual patient level showing a longitudinal series of events that tie into the time of biospecimen accessioning at a surgical diagnostic event. Each event at any level of the model is tagged with time points or time ranges.

Conclusion: The next steps are to receive pediatric data from PEDsnet in the same model as the simulation data inclusive of the large cohort of patients diagnosed with aggressive forms of cortical tumors as identified in our methods. The predictive models built in the exploratory analysis will be applied to the data as it is received. We intend to validate the data-driven phenotypes derived and apply their content as specimen annotations. Validation will occur by comparing well labeled data in the CBTTC with data derived from our models.

\section{Repository Management - LBA}

\section{LBP-8 Challenges in Integrating Automated Solutions for Biobanking Workflows: A Piece of Equipment is Not the Solution}

B. Trapp, M. Khan, R. Chakrabarty, H. Muppaneni, M. Derrig, A. Josey, T. A. Tran

Biorepository, Boston Children's Hospital, Boston, Massachusetts, United States 
The institutional biorepository at Boston Children's Hospital $(\mathrm{BCH})$ has applied instrumentation and informatics solutions to automate standard workflows. The challenges of implementing automation are not exclusive to the instrument, but in reality are multifaceted. A great deal of time, effort, and collaboration from all groups within the core is required, including informatics, management, research, and laboratory staff. Research and laboratory staff must standardize many procedures before a specimen can reach an instrument. Additionally, the informatics team must implement enhancements to the laboratory information management system (LIMS) to streamline the lab processes. The ability to overcome these challenges is the key to enabling innovation.

\section{LBP-9 Strategic Implementation to Establish Biorepository Sample Processing Platform in Clinical Laboratory for a Examination Remaining Blood Genomics Biobank Without Disrupting Clinical Examination Practice}

M. $\mathrm{Wu}^{1}$, K. Qian ${ }^{1,2}$, Y. Xiao ${ }^{1,3}$

${ }^{1}$ Department of Biological Repositories, Zhongnan Hopital of Wuhan University, Wuhan, Hubei, China, ${ }^{2}$ Laboratory of Precision Medicine, Zhongnan Hopital of Wuhan University, Wuhan, Hubei, China, ${ }^{3}$ Department of Urology, Zhongnan Hospital of Wuhan University, Wuhan, Hubei, China

Statement of the Problem: In the current era of individualized medicine, a large-scale genomics biorepository of human samples is essential to support clinical and translational research. Clinical laboratory collected and analyzed huge amounts of clinical blood, urine, fecal and clinical microorganism from health populations and patients. However, there are hurdles in the field of clinical laboratory and biobank, including workflow of examination remaining sample processing, safety and ethical issues.

Proposed Solution: To be familiar with the clinical laboratory workflow and management system, biobankers have a pre-job training in clinical laboratory. Standard operating procedures (SOPs) were drawn for examination remaining sample administration, sample collection, reception, temporary storage, processing, and shipping the samples to the Biorepository in accordance with the standards of ISO15189, ISO17025 and the ISBER Best Practices. With the goal of guaranteeing all the clinical waste should be safely disposed of in the clinical laboratory, a sample processing platform was established directly in clinical laboratory to support ethical issues counseling and sample processing. To ensure the examination remaining clinical sample should be temporarily stored at the cold storage for nearly 7 days to prevent re-examination without influence sample genomics quality, the examination remaining EDTA blood cell samples were processed in the clinical laboratory directly, then transported and stored at the Department of Biological Repositories (Zhongnan Biobank) according to the established SOPs under obtaining informed consents from individuals.

Conclusion: The implementation of establishing a biorepository sample processing platform in clinical laboratory has enabled us to validate a large amount of clinical laboratory examination remaining blood genomics biobank, and provide reliable strategy of cooperation with clinical and medical departments particularly in large population based disease cohort studies.

\section{LBP-10 The Biospecimen Research Database: An Online Literature Repository and SOP Library to Improve Specimen Quality}

L. D. Campbell, K. Engel, S. Greytak, E. Casas-Silva, P. Guan, H. M. Moore

\section{Biorepositories and Biospecimen Research Branch, National Cancer Institute, Bethesda, Maryland, United States}

This interactive demonstration will detail how to use the Biospecimen Research Database (BRD; biospecimens.cancer.gov/ brd) to locate standard operating procedures (SOPs) from a wide range of private, academic, and government institutions and to identify literature relevant to individual steps in preanalytical handling for specific analytes of interest. The BRD is a free and publicly accessible online tool from the National Cancer Institute (NCI)'s Biorepositories and Biospecimen Research Branch (BBRB) that contains more than 430 SOP documents submitted by over 60 participating sources and over 2700 peer-reviewed articles covering a broad range of topics spanning the biospecimen lifecycle, each with an original summary by a Ph.D.-level scientist. The presentation will demonstrate how to search the BRD's SOP library by keyword or curated fields (source organization, applicable biospecimens, and topic). Guidance will be provided on the creation of a session-specific compendium of SOP documents, allowing a user to view and download multiple SOPs as a zip-file rather than individually. The presentation will also include a demonstration on how to browse and submit tailored queries of literature entries for specific biomarkers or genes as well as preanalytical factors pertaining to biospecimen acquisition (collection method, timing, warm ischemia, etc.), preservation (type, duration, delivery method, etc.), storage (duration, temperature, freeze-thaw cycling, etc.), analyte isolation (method, washing, removal of protein, RNA or DNA, etc.), analytical methodology, and patient characteristics (age, gender, diagnosis, etc.). In addition, guidance for submitting article suggestions and SOP contributions through the website will be presented.

\section{LBP-11 A Unique Approach to Rare Disease Specimen Collection}

\section{Mann, G. Chery, B. Greenberg}

Neurology \& Neurotherapeutics, University of Texas Southwestern Medical Center, Dallas, Texas, United States

Background: Researchers in rare diseases face multiple challenges, including the availability of adequate numbers of quality biospecimens for study. Developing a method to collect research samples outside the clinic setting offers a way to increase numbers and promote diversity of the sample pool. To address this need for the University of Texas Southwestern Medical Center's open access biorepository for neuromyelitis optica (NMO), we established a staff position for a traveling research nurse whose focus is longitudinal collection of samples and data from patients with NMO.

Methods: The biorepository protocol was amended to allow collection and shipping of biospecimens outside the clinic setting. The travel nurse received certification in phlebotomy and IATA hazardous goods shipping. Biorepository lab personnel helped develop a protocol to standardize the collection, processing, and shipping of blood samples to be comparable to those collected on site. It was determined that the travel nurse could collect the same tube set as collected in clinic visits: 
ACDs shipped ambient, EDTAs and PaxGenes shipped refrigerated, and SSTs to be spun in a mobile centrifuge, aliquoted, and shipped on dry ice. The samples are shipped via FedEx to arrive at the lab within 24 hours of collection. The nurse recruited 40 subjects in 8 US locations to be visited 3 times per year. Visits are held in a private setting and take about 1 hour to collect, process, and pack each sample. Multiple visits may be scheduled in a day.

Results: Since 2014 we collected 266 samples from 45 research subjects with NMO outside our clinic. This compares to 285 samples from 58 subjects collected during clinic visits since 2010. Remote patients express appreciation that they may easily participate in research opportunities they might not otherwise have had. Quality and viability of the remotely collected samples are comparable to those collected in clinic. We have also been able to provide specialized collections for research collaborators including sharing DNA samples to understand the genetic underpinnings of this rare disease.

Conclusion: Use of traveling research personnel can augment the size and diversity of the biospecimen pool available for researchers in rare diseases. Also, the quality of the specimens is maintained through the shipping process. Lastly, patients feel more engaged in the research and are more likely to continuously contribute longitudinally.

\section{Biobanking Profiles - LBA}

\section{LBP-12 Setting \& Maintaining a Biobank with a Training Perspective in Laboratoire La Grace, Yaounde - Cameroon}

\section{J. M. Nana}

Diagnostic Medicine, Laboratoire d'Analyses Biomedicales La Grace, Yaoundé, Centre, Cameroon

Biobanks have been heralded as essential tools for translating biomedical research into practice, driving precision medicine to improve pathways for global healthcare treatment and services. There has been considerable investment in biobanking and research infrastructure in scientifically advanced countries. This is because of the perceived research benefits they provide. Setting, maintaining and managing biobanks is still a serious challenge in Cameroon. Virtually, no coherent strategy has been put in place to handle the challenges that the biobanking community has to handle. Biobanks present the interest of collecting both data and samples. Due to the advanced research in the field of HIV/AIDS and the emergence of antimicrobials resistance in Cameroon, many laboratories have started to constitute large biological collections, which unfortunately are more or less well preserved. The value of biological sample collection lies in the quality of the biological resources, their collection condition and also their preservation. The quality of the information associated with the samples being also very important. Yet, very few students, researchers or professionals are trained to the norms, the criteria and the processes of biobanks.

\section{LBP-13 National Liver Disease Biobank in India: Fostering Research Collaboration across India and Abroad}



${ }^{1}$ Biobank, National Liver Disease Biobank, New Delhi, India, ${ }^{2}$ Hepatology, Institute of Liver and Biliary Sciences, New Delhi, India, ${ }^{3}$ Department of Biotechnology, Government of India, New Delhi, India
Background: High Liver disease burden and diverse population in India provides an excellent opportunity to researchers. As per the World Health Organization liver disease is the tenth most common cause of death in India. The major causes of liver cirrhosis are Hepatitis B, C, alcohol abuse and non alcoholic fatty liver disease. There is very little progress has been done in this Field. Major obstruction in the translational research is unavailability of quality biosamples with standardized clinical annotations. Having a national level biobank and good research facility is the only solution to evade the problem. Institute of Liver and Biliary Sciences being a premier liver institute has established the National Liver Disease Biobank.

Methods: National Liver Disease Biobank proposal was submitted to central government agency for initial funding. The biobank was established in the area of 464.61 square meter at ILBS with a $\$ 3,531,713$ five-year grant from the Department of Biotechnology, Government of India. We have adopted decentralised sample collection, centralised storage and centralised informatics model for our national biobank facility. We sensitized the biobanking in different hospitals and research institutes by survey, brochures, phone calls and personal contacts to directors of hospitals and research institutes.

Results: Different committees are formed for smooth running of biobank. Biobank policies, consent forms in different languages, agreements forms and standard operating procedures are designed and evaluated by legal expert. We sensitized thirteen thousand scientists and doctors about biobanking concept and its scope in different hospitals and Institutes. We have signed MOU with two scientists and on term of talk with different hospitals and research institutes for signing of MOU for the sample collection and research. We have developed and are continuing to develop valuable relationships with general, hepatic and bariatric surgeons for sample collection.

Conclusion: Establishment of biobank is a major step in liver related clinical and basic research in India. It is a good platform to stimulate collaborations between clinicians and researchers, promoting multidisciplinary integration and advancing translational medicine to improve the health of Liver patients.

\section{LBP-14 Korea Biorepository Activity of Human Serum Bank for Infectious Disease Preparedness}

\author{
Y. Cha ${ }^{1}$, J. Kim ${ }^{1}$, J. Kim ${ }^{2}$, S. Lee ${ }^{2}$ \\ ${ }^{1}$ Chung-Ang University College of Medicine Seoul, Korea, \\ ${ }^{2}$ Chung-Ang University Hospital, Seoul, Korea
}

Background: As the globalization of the world, the infectious disease can spread very rapidly. To control infectious diseases, it can be necessary to develop vaccines, medicines, or in vitro diagnostic devices (IVDs). For supporting these infectious disease preparedness, Human Serum Bank (HSB) which is one of the leading Biological Resource Centers (BRCs) in South Korea collects human biological samples from infected patients or normal blood donors..

Methods: HSB collects blood samples in accordance with bioethical norms, maintains banking in an international quality management system and provides the high quality, accurately annotated human biological samples to the researchers, manufacturers, and quality control program operators in a fair distribution process.

Results: Since 2010, HSB has collected 194,860 human sera containing high risk viral antigens, antibodies or nucleic acids, 
such as HAV, HBV, HCV, or HIV $1 \& 2$ as well as normal blood donors, holding hundreds or thousands mother specimens according to the types of samples, and 74,325 (38.1\%) samples has been distributed to many different kinds of people who need them. Distributed human biological samples from HSB have been utilized mainly for the purpose of establishment, validation and verification of IVDs by manufacturers as well as basic research and also used for external and internal proficiency testing in hospital laboratories. HSB also has experiences for establishment of National Standards registered to Korea Ministry of Food and Drug Safety, especially anti-HIV-2 mixed performance panels being established recently.

Conclusions: HSB will contribute to the development of IVDs for the diagnosis and management of domestic infectious diseases and the expansion to the world by establishing a 'Global Bio-bank Network' for collection of rare samples.

Acknowledgement: The research resource was provided by the High-Risk Human Serum Bank (NRF-2017M3A9B8069581) through Bio \& Medical Technology Development Program of the MSIP, Korea.

\section{LBP-15 Challenges of Establishing a Human Cancer Biobank in a Developing Country: A Philippine Experience}

M. D. Enriquez, L. D. Cabral, I. Habaluyas, D. Chua-Ang, J. Andal, M. Imasa, R. Li

Human Cancer Biobank Research Center, St. Luke's Medical Center, Quezon City, Metro Manila, Philippines

The Human Cancer Biobank at St. Luke's Medical Center (SLMC) was conceived in 2014 by a team of oncologists, surgeons, pathologists and basic scientists with a goal of improving medical research in the country. It aims to provide scientists appropriate and adequate materials to study cancer as a prevailing health problem of the country. The team conceptualized the organizational structure, formulated the goals and policies, identified key partners in the hospital and worked for ethics approval of the project. In the beginning, four key challenges/needs were identified: (1) a considerable budget to cover purchase of necessary equipment, a software support for databanking, system of coding specimen, storage and retrieval of samples and salaries of the laboratory staff; (2) a committed group of clinicians and scientists who are willing to enrol subjects to the project; (3) well trained staff who will do data and sample collection as well as sample processing and storage; (4) a continuing system of improvement to make the whole project a sustainable. It was fortunate that SLMC one of the leading private tertiary hospitals in the country has a wellestablished research and biotechnology laboratory facilities; an accredited ethics and institutional review boards and seasoned researchers and clinicians who believe in the project. With the strong support from the hospital administration, the Human Cancer Biobank (HCB) of SLMC officially started its operation in January 2016 and has been marked the first functional cancer biobank in the country. At present, we have a total of 802 patients enrolled, from which the following samples were collected: liquid biopsies, fresh-frozen tissues, formalin-fixed paraffin embedded tissues as well as DNA and RNA derivatives. Top three cancer tissues collected are: breast, colon and thyroid. Problems and challenges continue to arise and we address these as best as we can. We look into improving our protocols, strengthen our translational research unit and build a research culture within our medical community. We hope that in the near future we can develop into a cross-sectional structure that will be able to work with other biobanks both locally and internationally. We are one in our belief that the establishment of this human cancer biobank is a major step towards establishing a national genomics research in the Philippines that will ultimately impact disease diagnosis, treatment and management.

\section{LBP-16 Management Mechanism of Clinical Specimen Bank in Large Comprehensive Hospital}

\section{S. Wang, X. Zhao, Y. Tian}

\section{Chinese People's Liberation Army General Hospital, Beijing, China}

As one of the largest comprehensive and first-class modern research-based hospital in China, Chinese People's Liberation Army General Hospital (PLAGH) has a great demand of clinical sample collection and storage in scientific research. Therefore, Clinical Specimen Bank (CSB) of PLAGH formulates a management mechanism to guide effective collection and rational usage of sample resources.

There are three sections in CSB, consisting of samples repository, data bank and resources sharing platform. samples repository stores and governs clinical samples. Data bank stores clinical information. Resources sharing platform releases information on sample description, disease categories, available quantity of storage and so on. Academic Committee, composed of professors and experts from laboratories or clinical departments of PLAGH, makes evaluation and verifies that the specimens are scientifically collected and used. Ethics Committee is responsible for the ethical examination of sample collection and usage. It also has the duty to supervise the informed consents signed and the right of privacy of patients protected.

As for the samples management, researchers who intend to collect and store specimens should submit an Application of Admission to CSB. Academic committee verifies the application materials and give permission. Then researchers sign an agreement with CSB on sample storage and administration. After the specimens are taken into the repository, all the related clinical information should be delivered to the Data Management System, to which different account numbers and authorities are set up and allocated. When the samples are required for using, researchers submit an Application of Releasing for permission and gain the samples conforming to the SOPs.

In order to assure sample quality, CSB tracks the procedure of sample collection and treatment by electronic information system. By consulting with patients, doctors obtain informed consent and give advices, which would be input to the Electronic Medical Record (EMR) and Health Information System (HIS). These processes make sure that the specimen information is correct and intact, and moreover the specimens are collected with normalization and legality.

With the working and development of the management mechanism, Clinical Specimen Bank of PLAGH plays an important role in promoting the achievements in scientific research and greatly improving the academic level and influence of PLAGH.

\section{LBP-17 China Biobank Profile}

X. Xu, B. Wang, P. Qian, X. Zhang, H. Lu, W. Zeng, Y. Shen, Q. Wan, J. Chen, Y. Shao

China National GeneBank, Shenzhen, Guangdong, China 
China National GeneBank (CNGB) is a non-profit organization supported by the Chinese Government.

CNGB is committed to develop a global biobank consortium to provide a platform for information sharing, Biobank materials exchanging, omics data acquisition, and Trans-omics scientific research. In order to contribute to the life science industry, CNGB has built an integrated infrastructure of "Three banks and Two platforms," that not only for bioresources and data storage, but also for data analysis and bioresources utilization.

Storage: The Biorepository of CNGB has stored 20 million traceable biological samples under the condition that complies with Quality Management Certification, and also establishes ultra-low temperature automatic storage system. CNGB launched a series of Specialized Biobanks, such as research cellbank, germplasm bank, crop germplasm bank, microorganism bank, population cohort bank.

The CNGB living biobank is committed to build China Noah's ark, which will protect and save life resources up to 300 thousand plant species, 1 million animal species, and 10 million microbe species.

Based on the massive data storage, CNGB has established over 48 databases, such as cancer database, millet database, covering over 7 thousand species, 27 individual species, 70,000 samples, 1 million genes, 10 million mutation information and 6 PB raw data. All these databases are free accessible to public, and all data can be retrieved from the CNGB website.

Reading: CNGB established a digitalization platform consisted of 200 self Research \& Developed BGI-seq500 sequencers, and one fully automated sequencer, revolocity. The annual output capacity of digitalization platform is $8 \mathrm{~PB}$, and capable of completing 500 human whole genome sequencing every year.

Writing: The synthesis and editing platform is the first and most advanced modular automation DNA synthesis platform in China. The platform successfully completed the synthesis of three yeast chromosomes, published 7 papers in the Science Special Issue, developed high efficient synthesis software, and set up standard laboratory procedure. CNGB applied 9 patents and 4 software copyrights through the platform and technology.

Global Collaboration: Through the Global Collaboration and Alliance department, CNGB will digitalize all global bioresources for better understanding of life, better utilization of the biological resources, better sharing of life science information, and better exploring future.

\section{LBP-18 PUMCH Biobank and National Rare Disease Registry System of China (NRDRS)}

D. Guo, Y. Jin, Z. Wu, Y. Xu, S. Zhang

PUMCH, Beijing, China

Background: As abundance clinical resources, Peking Union Medical College Hospital (PUMCH) has participated in National Science and Technology Specific Projects since 2012, and established a large-scale clinical biobank and database specialized on rare disease, malignant tumor, cardiovascular, metabolic diseases and so on. The biobank has had 8 categories of 89 diseases and Epidemiologic study as well as 50 thousand sets of specimens, that supported 63 items of research projects and more than high-quality 130 articles. The function of PUMCH Biobank has included support/service, scientific research, education/training, partnership/sharing.

Methods: As the diagnosis and guidance center which was appointed by Ministry of health, PUMCH focuses on rare dis- eases from diagnosis, treatment and research. With the low incidence, variety and difficult diagnosis, rare diseases have turned into an important medical problems for China and the world. Focusing on this issue, PUMCH take the led to establish the unified standard registration system for the national rare disease in China (NRDRS) to carry out large-scale registration and cohort research. To solve the resource scarcity problems, the project would set up cohort of 59 rare diseases with medical record and biospecimen, probe into study of the accurate phenotype and improve the understanding of the pathogenic mechanism of rare diseases. In December 2016, a publicity week was held for China national rare disease registration platform, and then launched international rare disease of China publicity week on February 2017, which with the theme of research brings infinite possibilities. Subsequently, project set up the rare diseases unit at PUMCH outpatient firstly, which began the patient registration, diagnosis, treatment and prognosis assessment. Biobank undertake the sample and medical record collection, biospecimen process, storage and sequencing. As the sponsor, NRDRS project has held the $12^{\text {th }}$ International Conference on rare diseases and orphan drugs (ICORD) in September 2017. All specialists, researchers and patient organizations of rare diseases joined the meeting and discussed the issues of drug development, research, policy and regulations, etc.

Results and Conclusion: As an important basis of translational medicine research, the value of biobank for rare diseases is fully embodied. The NRDRS project, which combining biobank and rare disease would bring new hope to patients and makes rare diseases rare no longer.

\section{Biospecimen Research and Science - LBA}

\section{LBP-19 Pin1 Promotes Regulated Necrosis Induced by Glutamate in Rat Retinal Neurons via CAST/calpain2 Pathway}

\section{S. Wang, J. Huang, K. Xiong \\ Central South University, Changsha, Hunan, China}

The purpose of the current study was to investigate whether peptidyl-prolyl cis/trans isomerase NIMA-interacting 1 (Pin1) can interact with calpastatin (CAST) and regulate CAST/ calpain2, under excessive glutamate conditions, and subsequently regulate necrosis in rat retinal neurons. Glutamate triggered CAST/calpain2-mediated necrosis regulation in primary cultured retinal neurons, as demonstrated by propidium iodidestaining and lactate dehydrogenase assay. Co-IP results and a computer simulation suggested that Pin1 could bind to CAST. Western blot, real-time quantitative polymerase chain reaction, immunofluorescence, and phosphorylation analysis results demonstrated that CAST was regulated by Pin1, as proven by the application of juglone (i.e., a Pin1 specific inhibitor). The retinal ganglion cell 5 cell line, combined with siRNA approach and flow cytometry, was then used to verify the regulatory pathway of Pin1 in CAST/calpain2-modulated neuronal necrosis that was induced by glutamate. Finally, in vivo studies further confirmed the role of Pin1 in CAST/calpain2-modulated necrosis following glutamate excitation, in the rat retinal ganglion cell and inner nuclear layers. In addition, a flash electroretinogram study provided evidence for the recovery of impaired visual function, which was induced by glutamate, with juglone treatment. Our work aims to investigate the involvement of the Pin1-CAST/calpain2 pathway in glutamate-mediated excitotoxicity. 
LBP-20 Immune-Checkpoints Screening in Tumor Specimens of Gastric Cancer from Hospital Biobank in Shanghai

Y. Yu ${ }^{1}$, J. Sun ${ }^{2}$

${ }^{1}$ Shanghai Institute of Digestive Surgery, Shanghai Ruijin Hospital, Shanghai, China, ${ }^{2}$ Gastroenterology, Ruijin Hospital, Shangjai Jiao Tong University School of Medicine, Shanghai, China

Background: The FDA approved Pembrolizumab targeting the programmed cell death 1 (PD-1) of lymphocytes for any unresectable or metastatic solid tumor with certain genetic anomalies such as microsatellite instability-high (MSI-H) or mismatch repair deficient (dMMR) biomarker. This approval marked the first instance in which the FDA approved marketing of a drug based only on a common biomarker rather than tumors in a specified location. It was reported that several types of malignancies were disclosed by MSI-H status due to dMMR and characterized by increased neoantigen load to elicit intense host immune in the tumor microenvironment.

Methods: In this study, we screened the PD-1/PD-L1 expression, EB virus and MSI status in a large cohort of gastric cancer deposited in hospital biobank.

Results: We clarified the proportion of main immunecheckpoints PD-1/PD-L1, positive rate of EB virus infection and MSI status in tumor specimens of gastric cancer and its relationships with clinicopathological variables.

Conclusions; The preliminary data revealed its potential value for selecting proper patients for new immune therapy.

\section{LBP-21 Comparing the Immune Profile of NMO Patients Receiving Mechanistically Distinct Immunomodulatory Therapies}

O. Anibire, G. Chery, R. Harbert, B. Greenberg, N. Monson

Neurology, University of Texas Southwestern Medical Center, Dallas, Texas, United States

Neuromyelitis Optica (NMO) is a rare, autoimmune, inflammatory demyelinating disease that affects both the spinal cord and optic nerves. Although there is no cure, immunosuppressive drugs such as Rituximab and Mycophenolate Mofetil (MMF) are wellknown management strategies used to treat this disorder. Rituximab is an antibody that specifically targets CD20 proteins expressed on the surface of B cells which are a cell type of the immune system that contributes to the pathology associated with NMO. MMF is also used to treat NMO, but was originally used as an immunosuppressant drug to reduce the immune response towards the transplanted organs. However, there have been no studies to determine whether the immune cell profiles are altered in response to MMF in NMO patients. The aim of this study is to compare the concentration of PBMCs in the NMO patient cohort on Rituximab and the NMO patient cohort on MMF. The second comparison was between each patient cohort and the age- and sexmatched healthy donor cohort. Blood was obtained in Acid Citrate Dextrose (ACD) tubes from 12 NMO patients on Rituximab, 12 patients on MMF and 12 age- and sex-matched healthy controls. ACD tubes were spun in a centrifuge at 1400 revolutions per minute (RPM) for 10 minutes with the break off. Upon completion of the spin, plasma was extracted from the samples which was aliquoted and stored for future research. To obtain isolated peripheral blood mononuclear cells (PBMCs), the remaining blood was diluted at a 1:1 ratio with room temperature $1 \mathrm{X}$ Phosphate Buffered Saline (PBS), layered over Ficoll-Hypaque and then spun at 1900 RPM for 25 minutes with the break off. The first gradient containing plasma/PBS was discarded while the second layer containing the PBMCs was extracted and was later used to determine the concentration of PBMCs per $\mathrm{mL}$ of blood. This study is currently on going, however, the data obtained will be presented during the ISBER conference this spring

\section{LBP-22 Clinicopathological Significance and Prognostic Role of EZH2 Expression in Pulmonary Adenocarcinoma with a Micropapillary Pattern}

A. Wang, D. Guo, X. Zhao, Q. Xie

\section{PUMCH, Beijing, China}

Background: Lung adenocarcinoma as a common histological type of lung cancer, accounting for about $50 \%$ of lung cancer. Pulmonary adenocarcinoma with a micropapillary pattern (MPPAC) was briefly described in the WHO (2004 edition) histological classification of lung cancer, due to its unique molecular biological characteristics, highly invasive mechanism and poor prognosis, it has attracted more and more attention from domestic and foreign scholars.

Methods: Our study collected 84 cases of MPPAC specimens from the Department of Pathology Biobank, PUMCH from 2004 to 2015 , with MPP $\geq 50 \%$ and complete medical history. The tissue microarray of 84 cases were stained with immunohistochemistry (IHC), and the expressions of enhancer of zeste homolog2(EZH2) were divided into high and low expression groups. Then we analyzed the relationship between EZH2 expression and clinicopathological features and prognosis with chi-square test. The survival curves were analyzed with 58 patients $(72.7 \%)$ using the KaplanMeier method, and $\mathrm{P}$ values were calculated using the log-rank test, with $\mathrm{P}<0.05$ being considered of statistical significance. Multivariate Cox risk regression analysis was used to assess the relationship of lymph node metastasis, EZH2 expression, tumor diameter and other factors with prognosis.

Results: The study showed that the expression of EZH2 only associated with smoking history $(\mathrm{P}=0.030)$, which was no association with other factors such as TNM stage $(\mathrm{P}=0.348)$, lymph node metastasis $(\mathrm{P}=0.403)$, age $(\mathrm{P}=0.875)$, gender $(\mathrm{P}=0.782)$, tumor size $(\mathrm{P}=0.967)$. The survival time of EZH2 high and low expression groups was analyzed by Kaplan-Meier method, and the data showed that the survival time of EZH2 low expression group was longer than high expression group, and the median survival time of patients each with high and low expression group were $43 \pm 5.853$ and $99 \pm 24.074$ months. But there is no significant statistical differences between two groups through the analysis of log-rank test $(\mathrm{c} 2=2.961, \mathrm{P}=0.085)$. Multivariate Cox risk regression analysis showed that lymph node metastasis was an independent risk factor for MPPAC patients, and a Cox model survival curve showed that this risk would lead to an increased risk of death.

Conclusions: The results showed that EZH2 expression of patients with poor prognosis worse, which indicating that EZH2 is expected to become a new prognostic indicators of MPPAC patients.

\section{LBP-23 High-Quality Samples for Biobanking - Biobank Graz Concept for Quality Assurance and Quality Control}

F. Vogl ${ }^{1}$, K. Plattner ${ }^{1}$, M. Bayer ${ }^{1}$, K. Sargsyan ${ }^{1,2}$

${ }^{1}$ Biobank Graz, Medical University Graz, Graz, Austria,

${ }^{2}$ Yerevan State Medical University, Yerevan, Armenia 
Statement of Problem: Biobanks have a pivotal role in biomedical research by providing samples and data. In the last years, sample analysis technologies have become more sophisticated and concomitantly sample quality became more and more important. Nevertheless, researchers complain about an inadequate access to quality biospecimens.

Proposed Solution: Biobank Graz, one of the largest repositories of clinical samples in Europe, stores more than 7.5 million biospecimens and their associated data covering 30 years of collection. Therefore, these samples are very useful for long-term quality studies. Here, we present a concept for quality assurance and quality control in biobanking and discuss different sample analysis services for various quality biomarkers.

In biobanking, the preanalytical phase (including sample collection and transportation) is the most critical and errorprone part. When samples are finally stored in a biorepository, mainly storage conditions and duration have a huge impact on sample quality.

First, it is important to define activities that ensure the maintenance of biospecimen quality (quality assurance; QA). This includes the implementation of the CEN standards. In the second step, biobanks need to implement reliable techniques and tools to evaluate their actual sample quality (quality control; QC).

For the latter, a wide range of standardized and validated sample analysis methods are available. QC tools can focus on morphology, on data quality or on biomolecules. The latter comprises nucleic acids (DNA, RNA), proteins and metabolites. Histologic quality control involves morphological validation of tissue. For biomedical research, also access to high-quality sampleassociated data is crucial. Finally, QC activities depend on the material (e.g. tissue or blood) and on the analyte of interest.

Conclusion: Quality control in a biorepository encloses QC of analytes/biomolecules, morphological QC and data quality:

a) Biomolecules: different methods/assays and quality biomarkers

b) Morphology: morphological validation of tissue samples

c) Data: high-quality pathological and clinical data required

In biobanking it is not always possible to control all variables (especially in the preanalytical phase), but their documentation is important. Finally, the overall goal of QA and QC in biobanking is to provide fit-for-purpose biospecimens for researchers.

\section{LBP-24 Association Between STAT4 Polymorphisms and the Risk of Juvenile Idiopathic Arthritis in Han Chinese Populations}

X. Huang, N. Jia, J. Zou, X. Cui

Capital Institute of Pediatrics, Beijing, China

Background: Juvenile idiopathic arthritis (JIA) is a chronic autoimmune disease, and characterized by arthritis more than 6 weeks in children less than 16 years. Previous studies have already demonstrated potential role of STAT4 polymorphisms and JIA risk in Caucasian populations, while this impact in Han Chinese populations remains unclear. The purpose of this study was to investigate the single nucleotide polymorphisms (SNPs) of STAT4 and JIA in Han Chinese populations according to disease types.

Method: A total of 205 JIA cases and 267 healthy controls were included in this study, and 17 selected SNP sites in the STAT4 gene were analyzed with MassArray high-throughput DNA analyzer with matrix-assisted laser desorption/ionization time-of-flight mass spectrometry. Multiple logistic regressions were employed to calculate the relationship between the SNPs of STAT4 and the risk of JIA.

Results: The G allele of rs11893432 was associated with an increased risks of total JIA (odds ratio [OR]: 1.73 ; 95\% confidence interval $[\mathrm{CI}]: 1.03-2.88 ; \mathrm{P}=0.037)$. This significant relationship was main focused on oligoarticular JIA (OR: 2.75; 95\% CI: 1.29-5.83; $\mathrm{P}=0.026$ ), while it was not affect the incidence of polyarticular JIA or systemic JIA. Subjects carrier GG was significantly correlated with oligoarticular JIA risk as compared with subjects carrier $\mathrm{CC}+\mathrm{CG}(\mathrm{OR}$ : 1.88; 95\% CI: 1.06-3.32; $\mathrm{P}=0.034)$. In addition, the $\mathrm{C}$ allele of rs1018981 and A allele of rs10931481 were associated with greater risk of polyarticular JIA (C allele: [OR: 7.82; 95\% CI: 1.06-57.74; $\mathrm{P}=0.044$ ]; A allele: [OR: 2.86; 95\% CI: 1.23, 6.65; $\mathrm{P}=0.039$ ).

Conclusions: The SNP of G allele in rs11893432 of STAT4 was closely related to JIA, particularly oligoarticular JIA in Han Chinese populations. The $\mathrm{C}$ allele in rs1018981 and $\mathrm{A}$ allele in rs10931481 were correlated with the risk of polyarticular JIA.

\section{LBP-25 Study of the Potential Tumor Markers of Renal Clear Cell Carcinoma with Proteomic}

X. Zhao, Q. Xie, B. Long, X. Xu, D. Guo

PUMCH, Beijing, China

Background: Renal cell carcinoma (RCC) is the most frequent neoplasm of the kidney, and its morbidity is around 3\% in malignant tumor. Despite significant progress that has been made in the treatment of metastatic RCC, nephrectomy remains the only effective treatment for localized RCC. However, the recurrence rate of operation is significant, more than $30 \%$ of the patients have distant metastasis following surgery, and high rate of resistance against chemotherapeutic agents and radiation therapy make the therapeutic options for these patients is very limited. We try to identify and validate the potential tumor markers of renal clear cell carcinoma for progress assessment and clinical treatment with proteomic.

Methods: We analyzed 16 cases tumor and Pericarcinomatous tissue with 2D-DIGE and Q-Fourier transform ion cyclotron resonance mass spectrometry (FT-ICR MS), and then identified the abnormal expression protein with Western blot, Real time PCR and IHC in 84 patients of 100 paired tissues.

Results: A total of 1873 proteins were quantified with proteomic in 16 paired Renal clear cell carcinoma, and 51 proteins of 68 sports that more than 1.5 -fold differential expressing were identified, including 16 proteins up-regulated and 35 proteins down-regulated in RCC $(\mathrm{p}<0.05)$. The proteins contain ion channel activity, transport activity, enzyme regulation activity, catalytic activity, structural molecule, binding and transfer function. Among them, there were two upregulated NNMT, ANXA4and down-regulated ACY1, Calbindin, which were confirmed with 16 paried tumor and pericarcinomatous tissues with Q-PCR and western blot $(\mathrm{p}<0.01$ or $\mathrm{p}<0.001)$. Then the SPSS analysis of IHC validated ACY1 were decreased in 53 cases of ccRCC ( $<<0.001)$, and ANXA4 were increased in 47 cases $(\mathrm{p}<0.001)$, and the IHC differently expression of ACY1 and ANXA4 had no relation with demographic features(Age/Sex), clinical pathology (Stage/Grade), living style(Drinking/Smoking) and other disease (Diabetes/ Hypertension).

Conclusion: ACY1 and ANXA4 could be serve as putative tumor suppressor, which appears as a potential treatment marker for clear cell RCC would be further studied. 
LBP-26 The Results of Peripheral Blood Mononuclear Cells Isolated from Liver Disease and HIV-1 Infected Individuals from Hepatitis/AIDS Biobank

\section{J. Sun, H. Sun}

Beijing Youan Hospital, Capital Medical University, Beijing, China

Background: The quality of isolated peripheral blood mononuclear cells (PBMC) can affect the preservation of PBMC, thus affecting subsequent scientific research. Liver disease and HIV-1 infected individuals are the main study population in Hepatitis/AIDS Biobank. Analysis the PBMC of this two groups could help to better control the quality of frozen PBMC.

Methods: 121 samples from Hepatitis/AIDS Biobank including 35 liver disease, 51 HIV-1 infected individuals and 35 control, according the diagnosis were analyzed. Ficoll density gradient centrifugation to separate $\mathrm{PBMC}, \mathrm{AO} / \mathrm{PI}$ double staining method to detect the living cells, total cell and cell viability of PBMC.

Results: The total cells results show that the control $(1.54 \mathrm{E}+06,9.03 \mathrm{E}+05 \sim 3.53 \mathrm{E}+06)$ were highest, followed by HIV infected $(1.08 \mathrm{E}+06,3.72 \mathrm{E}+05 \sim 2.82 \mathrm{E}+06)$, finally for liver disease $(8.49 \mathrm{E}+05,3.61 \mathrm{E}+05 \sim 2.30 \mathrm{E}+06, \mathrm{p}<0.05)$. Living cells results show that the control group were the highest $(1.53 \mathrm{E}+06,8.79 \mathrm{E}+05 \sim 3.51 \mathrm{E}+06)$, followed by HIV-1 infected individuals $(1.06 \mathrm{E}+06,3.46 \mathrm{E}+05 \sim 2.77 \mathrm{E}+06)$, finally for liver disease $(8.44 \mathrm{E}+05,3.54 \mathrm{E}+05 \sim 2.25 \mathrm{E}+06, \mathrm{p}<0.05)$. The cell

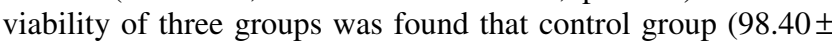
$0.39)$, higher than that in liver disease (97.28 \pm 0.33 ) and HIVinfected (96.86 \pm 0.37$)$, with a statistically significant difference $(\mathrm{p}<0.05)$.

Conclusions: the isolation of PBMC from liver disease and HIV infected were affected, the number ant the cell viability of PBMC was lower than that of control group.

\section{LBP-27 Role of Aldehyde Dehydrogenase 1A2 in Ovarian} Cancer

H. Kwon ${ }^{1,2}$, J. Choi ${ }^{1,2}$, J. Kim ${ }^{1,2}$

${ }^{1}$ Obstetrics and Gynecology, Gangnam Severance Hospital, Biomedical Research Center, Seoul, Korea, ${ }^{2}$ Obstetrics and Gynecology, Gangnam Severance Hospital, Yonsei University College of Medicine, Seoul, Korea

Aldehyde dehydrogenase (ALDH), retinoic acid synthesis gene, is an enzymes for detoxification of endogenous and exogenous aldehyde substrates through NAD $(\mathrm{P})+$-dependent oxidation. Recent evidence suggests that enhanced ALDH activity is a measurable hallmark of cancer stem cells. However, we found the expression of aldehyde dehydrogenase 1 family, member A2 (ALDH1A2), is significantly decreased in ovarian cancer tissues compared to normal ovarian tissues. Low ALDH1A2 expression was implicated with an unfavorable prognosis of ovarian cancer patients, suggesting the possibility that ALDH1A2 have a critical role as a tumor suppressor gene in ovarian cancer. Furthermore, we observed that the forced expression of ALDHA2 gene impaired significantly cell proliferation and invasive activity via time-dependent manner in ovarian cancer. A small interfering RNA (siRNA) targeted against ALDH1A2 resulted in accelerate colony formation in soft agar in human ovarian surface epithelial (HOSE) cells. Collectively, our findings reveal that ALDH1A2 is a candidate tumor suppressor, further suggesting that the enhanced ALDH1A2-linked pathway might provide new opportunities for therapeutic intervention in ovarian cancer.

\section{LBP-28 Exploring the Improvement of Cryopreserving Human Peripheral Blood Mononuclear Cells}

H. Guo, Y. Xu, A. Zang, Q. Zou, J. Zhang, Y. Xu, L. Yao, X. $\mathrm{He}$

\section{Origincell Technology Group Co., Ltd., Shanghai, China}

The cryopreservation of human peripheral blood mononuclear cells (PBMCs) is a critical issue left non-elucidated thoroughly in the field of bio-preservation. Many factors remain to be examined about their impacts on the quality of PBMC cryopreservation. To address this issue, we carried out a systematic investigation of relevant factors involved in every step of cryopreservation from the initial leuko-apheresis, subsequent traffic, freezing and thawing of PBMCs.

Upon the leuko-apheresis, the contamination of red blood cells (RBCs) other than platelets was likely to adversely affect the cell viability and cell recovery of frozen PBMCs when its concentration was more than $0.5 \times 10 \mathrm{e} 12 \mathrm{cell} / \mathrm{L}$. Gentle vibration rates lower than $200 \mathrm{rpm} / \mathrm{min}$ resulting from traffic didn't influence the subsequent cryopreservation of PBMCs, while the vibration of more than $1500 \mathrm{rpm} / \mathrm{min}$ led to a dramatic reduction of cell viability and cell recovery. After that, fresh PBMCs can be stored at $4-8{ }^{\circ} \mathrm{C}$ for at least $48 \mathrm{~h}$ without any loss of cell viability for subsequent cryopreservation. Then, we investigated the optimal cell concentration verse volume of cryomedium in each vial, formulation of cyromedium in the presence of DMSO as the cryoprotectant, as well the operational fashions. For a cryovial of $2.0 \mathrm{ml}, 1 \times 10 \mathrm{e} 7$ cells in $1.5 \mathrm{ml}$ cryomedium was the best option, having near $95 \%$ cell viability while the number reduced to less than $90 \%$ when the concentrations were higher than 5X10e7. By developing a cyromedium, we obtained the better quality of frozen PBMCs than those by using commercial cyromedium. And thawing cells at $42{ }^{\circ} \mathrm{C}$ or $65^{\circ} \mathrm{C}$ contributed to an increasing improvement of cell viability and cell recovery than $37{ }^{\circ} \mathrm{C}$. In conclusion, newly defined key factors here not only improved our understanding of PBMC cryopreservation, but also would significantly contribute to the standardization of PBMC bio-banking worldwide.

\section{LBP-29 Optimizing Thawing Temperature to Improve the Quality of Cryopreserved Human Peripheral Blood Mononuclear Cells}

Y. Xu, H. Guo, A. Zang, D. Wang, S. Liu, S. Xue, J. Huang, X. He

\section{Origincell Technology Group Co., Ltd., Shanghai, China}

Thawing is an important step in cryopreservation, the conversion of cells from a frozen, glassy state where they exist in a stasis, to a viable temperature where on recovery they are able to proliferate. However, literature regarding the thawing of cryopreserved human peripheral blood mononuclear cells is surprisingly limited and therefore an apparent lack of understanding remains. Protocols usually advise rapid warming in a $37^{\circ} \mathrm{C}$ water bath. Nevertheless, it is essential for more definition of the critical parameters in thawing process, especially for the temperature used to de-freeze frozen PBMCs.

Our present findings uncovered the fact that using $42{ }^{\circ} \mathrm{C}$ or $65^{\circ} \mathrm{C}$ as alternative thawing temperature instead of regular $37^{\circ} \mathrm{C}$ led to an increasing improvement of cell viability and cell 
recovery for PBMCs and two cell lines, i.e. 7721 and McAb. Then, we performed the phenotypic and functional analysis to further assess the consequence of thawing at elevating temperature. It turned out that elevating thawing temperature did not impair the population of $\mathrm{T}, \mathrm{B}, \mathrm{NK}$ cells in PBMCs, and PHA-induced $\mathrm{T}$ cell responses including the cell surface expression of activation marker CD69 and CD25 as well as the expansion capacity in LPA assay. Nevertheless, PHA-triggered release of IFN $\gamma$ and IL-2 was much higher in $65^{\circ} \mathrm{C}$-thawed PBMCs than those in $42{ }^{\circ} \mathrm{C}$ and $37{ }^{\circ} \mathrm{C}$. These data strongly demonstrated the most protective capacity of using $65^{\circ} \mathrm{C}$ as thawing temperature for PBMC instead of $37^{\circ} \mathrm{C}$.

\section{LBP-30 Molecular Biological Characterization of Newly Established Epithelial Ovarian Cancer (EOC) Cell Lines}

H. Kwon ${ }^{1,2}$, E. Lee ${ }^{1,2}$, W. Yang ${ }^{1,2}$, H. Shin ${ }^{1,2}$, J. Choi ${ }^{1,2}$, J. $\operatorname{Kim}^{1,2}$

${ }^{1}$ Obstetrics and Gynecology, Gangnam Severance Hospital, Biomedical Research Center, Seoul, Korea, ${ }^{2}$ Obstetrics and Gynecology, Gangnam Severance Hospital, Yonsei University College of Medicine, Seoul, Korea

Background: Human biospecimens are critical in accelerating development of molecular-based diagnostics and therapeutics for precision medicine.

Human biospecimens, such as tissue, blood, urine and saliva have emerged as critical resources for basic and translational research in gynecologic cancer because they are the direct sources of molecular mechanism research used in the initiation of a tumor, and its progression for metastatic disease and resistance for treatment. However, its application for research use has been limited. Recently immortalized cell lines are widely used in further personalized medicine customized to the study. Experimental models such as immortalized cancer cell lines are very useful tools because they can serve as substitutes for in vivo human body conditions through in vitro experiments.

So we report the characterization of 5 immortalized cell lines (designated YDOV-13, YDOV-139, YDOV-151, YDOV-157 and YDOV-161) established from primary tumor samples of Korean patients.

Materials and Methods: The immortalized cell lines were established in 2005 to 2006 from Primary tumor tissues of a $20 \sim 70$ year-old women presenting with ovarian cancer. Five newly established EOC cell lines were characterized by transcript profiling. Microarray analyses were validated using quantitative RT-PCR.

Results: A total of nine immortalized cell lines derived from Korean patients were established in 199 and MCDB 105 (1:1) medium. Population doubling times ranged from 197-133 h and cell viability after thawing was about $85 \%$. All immortalized cell lines were free of contamination from either bacteria or mycoplasma. A total of 239 genes represented by 24,957 probes were identified from microarray analysis, of which 98 and 141 genes are up- $(>2$ fold, $\mathrm{p}\langle 0.05)$ or down-regulated $(\langle 3$ fold, $\mathrm{p}\langle 0.05)$, respectively, in EOC cell line compare to human ovarian surface epithelial cell lines. We identified a subset of EOC characterized by the numerous genes related to cell cycle and apoptosis.

Discussions: Much of the current knowledge on biology of gynecologic cancer is based on in vivo and in vitro studies performed with immortalized cell lines. These well-characterized immortalized cell lines will be useful for the study of gynecologic cancer biology.
The newly derived immortalized cell lines may be an important research resource for studying cancer cell biology and should also be very useful for developing new strategies that inhibit cancer cell growth and progression.

\section{LBP-31 Temporal RNA Integrity Analysis of Archived NASA Spaceflight Biological Samples - 1991 to 2016}

E. Delgadillo ${ }^{1}$, A. French ${ }^{2}$, D. Lopez ${ }^{2}$, S. Lai-Polo ${ }^{3}$, V. Boyko ${ }^{3}$, M. Dinh ${ }^{3}$, J. Rask ${ }^{2}$, K. Chakravarty ${ }^{4}$, H. Stewart ${ }^{1}$

${ }^{1}$ Ames Life Science Data Archive, NASA, Moffett Field, California, United States, ${ }^{2}$ Ames Life Science Data Archive, NASA/WyLe, Moffett Field, California, United States, ${ }^{3}$ GeneLab, NASA/WyLe, Moffett Field, California, United States, ${ }^{4}$ Ames Life Science Data Archive, NASA/Logyx LLC, Moffett Field, California, United States

The purpose of this study is to assess the quality of NASA spaceflight tissues stored in Ames Life Science Data Archive (ALSDA) -80C freezers. Garnering information - for downstream functional analysis such as generation of omics datasets - from tissues is, in part, dependent on the state of sample preservation. To assess the viability of a select group of tissues, RNA integrity number (RIN) values were calculated for RNA extracted from rodent livers. Rat livers from Spacelab Life Sciences 1 (SLS-1) and mouse livers from Commercial Biomedical Test Module 3 (CBTM-3), Rodent Research 1 (RR1), and Rodent Research 3 (RR3) were tested. It was found that mean RIN values from CBTM3, RR1, and RR3 were suitable for downstream functional analysis (RIN >5) while the mean RIN value for SLS-1 was not (RIN=2.5 \pm 0.1$)$. Information from this study could lay the foundation for future efforts in determining the types of assays that are most appropriate for different tissues stored in ALSDA -80C freezers, therefore maximizing the scientific return on rare spaceflight samples.

\section{LBP-32 Effect of Using Previously Banked Hair Samples on SNP Genotyping of Livestock Animals}

\section{A. A. Zwane}

Animal Breeding and Genetics, Agricultural Research Council, Pretoria, Gauteng, South Africa

In the past years, genome-wide single nucleotide polymorphisms (SNPs) have become increasingly popular as a marker of choice in population genetic studies. Genetic sampling methods, which includes hair, tissue, and blood samples among others, has increased the scope and efficiency of genomic studies in livestock populations such as cattle, sheep, goats, and pigs. Many previously banked biomaterials have been unsuitable for DNA analyses due to the extensive degradation (Der Sarkissian et al., 2015) due to poor sampling and storage conditions. Environmental exposure to moisture and ultraviolet radiation, along with collection and storage methods, can degrade DNA in biological samples, whereas cool and dry conditions are known to minimize degradation (Stetz et al., 2015). Sequences of DNA can be easily broken as DNA is a fragile molecule (Lindahl 1993), which in turn can affect SNP genotyping. As genotyping is still expensive in most developing countries, lower call rate and failed genotyping can be a challenge since repeated genotyping is costly. Therefore, limiting exposure to DNA degradation on collected samples is 
important component for genomic study design. This study shows the effect of using previously banked cattle hair samples on SNP genotyping, and how the sampling and storage methods can be improved for better quality DNA. Twenty-four cattle hair samples, collected between the years 1997 and 2012 were retrieved form the ARC Biobank. Several hair roots were cut to extract DNA using phenol and chloroform protocol. Samples were genotyped using bovine $150 \mathrm{~K}$ assay on an Illumina platform. DNA quantification showed the ratio of UV absorbance ranging from 1.5 to 2 , showing the quality of DNA. From the total genotyping, approximately only $58 \%$ of samples were successfully genotyped with a lower proportion of sample with low genotyping call rate. This shows that there was a notable level of degradation on selected samples. This could be due to the condition from which samples were collected, part of the animal body from which hair samples were plucked, or the storage condition after collection. Therefore, proper sampling and storage methods for hair samples must be considered to preserve high quality DNA sufficient for genomic studies.

\section{LBP-33 Characterizing Freezing Responses of Human iPS Cells Using Low-Temperature Raman Spectroscopy}

G. $\mathrm{Yu}^{1}, \mathrm{R} \cdot \mathrm{Li}^{2}$, A. Hubel ${ }^{1}$

${ }^{1}$ Mechanical Engineering, University of Minnesota, Saint Paul, Minnesota, United States, ${ }^{2}$ Biomedical Engineering, University of Minnesota, Minneapolis, Minnesota, United States

Background: Human induced pluripotent stem cells (hiPSCs) have attracted much research interests in tissue engineering and personalized medicine. Effective cryopreservation of hiPSCs is required for both clinical and scientific purposes. In this study, Raman spectroscopy was used to characterize the freezing responses of both hiPSC single cells and aggregates cryopreserved in DMSO solution at three cooling rates $\left(1,3\right.$, and $\left.10^{\circ} \mathrm{C} / \mathrm{min}\right)$ and two seeding temperatures $\left(-4\right.$ and $\left.-8^{\circ} \mathrm{C}\right)$. Freezing responses of hiPSC aggregates cryopreserved in a combination solution of sucrose, glycerol and isoleucine at seeding temperature of $-4^{\circ} \mathrm{C}$ and cooling rate of $1{ }^{\circ} \mathrm{C} / \mathrm{min}$ were also investigated as there is demand for DMSO-free cryoprotectants that maintain cell viability and function.

Methods: Raman Spectroscopy measurements were conducted using WITec Confocal Raman Microscope System Alpha $300 \mathrm{R}$ with $532 \mathrm{~nm}$ wavelength laser as the excitation source. Raman images were assembled by integrating the spectrum at each pixel based on characteristic wavenumbers of common intracellular and extracellular material. Sample temperature was controlled using a four-stage Peltier and a series-800 temperature controller to a final temperature of $-50^{\circ} \mathrm{C}$ using the desired cooling rate and seeding temperature.

Results: For single cells and aggregates cryopreserved in DMSO solution, Raman images of ice showed that lower seeding temperature $\left(-8^{\circ} \mathrm{C}\right)$ did not affect IIF in single cells but significantly increased IIF in aggregates, suggesting higher sensitivity of aggregates to supercooling. Higher cooling rate $\left(10^{\circ} \mathrm{C} / \mathrm{min}\right)$ resulted in more IIF in single cells at both seeding temperatures and in aggregates at seeding temperature of $-4^{\circ} \mathrm{C}$. Raman images of DMSO showed greater variation of DMSO concentration across aggregates than single cells, suggesting cryoprotectant transport limitations in aggregates.

Cell aggregates cryopreserved in the osmolytes solution at seeding temperature of $-4^{\circ} \mathrm{C}$ and cooling rate of $1{ }^{\circ} \mathrm{C} / \mathrm{min}$ showed similar amount of IIF compared to those frozen in DMSO solution, suggesting effective IIF prevention of these osmolytes. However, there was a larger gradient of osmolytes concentration across the aggregates than that of DMSO concentration, indicating greater transport limitations of osmolytes in aggregates.

Conclusion: Taken together, the results from these studies improve our understanding of DMSO and non-DMSO cryoprotectants solutions and the manner by which they affect the freezing responses of hiPSCs.

\section{LBP-34 Extracting DNA from Formalin-Fixed, Paraffin- Embedded (FFPE) Tissue Biospecimens Using User-Friendly Automated Technology: Is There an Impact on Yield or Quality?}

W. Mathieson ${ }^{1}$, N. Guljar ${ }^{2}$, I. Sanchez ${ }^{1}$, M. Sroya ${ }^{2}$, G. A. Thomas ${ }^{2}$

${ }^{1}$ Integrated Biobank of Luxembourg, Luxembourg, ${ }^{2}$ Imperial College London, London, United Kingdom

Background: Protocols to extract DNA from FFPE biospecimens are often long and complex, so automation is an attractive option when large numbers of biospecimens need processing or technicians are less experienced. It is unclear whether automation yields poorer DNA than manual extractions performed by experienced technicians. We compared manual extractions, manual deparaffinization/automated DNA extractions and a platform designed for user-friendliness in which DNA extraction is fully automated, linking seamlessly to semi-automated deparaffinization.

Methods: Four $10 \mu \mathrm{m}$ sections were cut from 42 clinical FFPE biospecimens, then 1 section used per extraction method. In both manual and semi-automated extractions, deparaffinization was carried out manually (Xylene then ethanol) then DNA extracted using the QIAamp FFPE kit (Qiagen), either manually or using a QiaCube (Qiagen). Fully automated extractions were performed using the ExScale Biospecimen Solutions platform; deparaffinization was carried out automatically after the tissue sections were centrifuged in ExScale Deparaffinization Solution. ExScale extractions were performed using two kits: one extracting DNA only (ExScale DNA only) and the other simultaneously extracting DNA and RNA (ExScale simultaneous). DNA was quantified using Pico Green and its integrity assessed using the qPCR-based Illumina FFPE QC Assay and nano electrophoresis-derived DNA Integrity Numbers (Agilent 4200 TapeStation running Genomic DNA ScreenTapes).

Results: DNA extractions were successful in 91\% (manual), 93\% (QiaCube), 81\% (ExScale DNA only) and 74\% (ExScale simultaneous) biospecimens. Median yield (ng/section) was 385 (manual), 300 (QiaCube), 260 (ExScale DNA only) and 160 (ExScale simultaneous). In the Illumina FFPE QC Assay, lower delta $\mathrm{Cq}$ values denote better quality DNA. Median delta $\mathrm{Cq}$ was 0.1 (Manual), 0.6 (QiaCube), 1.2 (ExScale DNA only) and 1.0 (ExScale simultaneous). DINs range from 1-10, with higher DINs equating to higher quality DNA. Median DINs were 6.0 (manual), 5.7 (QiaCube), 5.7 (ExScale DNA only) and 4.7 (ExScale simultaneous).

Conclusion: DNA extracted manually by skilled technicians had highest yield and integrity. ExScale was the fastest and required the least intervention or technician experience, but returned poorest DNA yield and quality. The semi-automated QiaCube extractions were intermediate. Thus, the most appropriate method depends on technician experience and the numbers of samples to be processed. 


\section{LBP-35 Quality of Plasma from Barricor Blood Collection Tubes}

C. Mathay ${ }^{1}$, N. Schwartz ${ }^{2}$, E. Henry ${ }^{1}$, M. Marchese ${ }^{1}$, M. Kiehntopf ${ }^{2}$, F. Betsou ${ }^{1}$

${ }^{1}$ Integrated BioBank of Luxembourg, Dudelange, Luxembourg, ${ }^{2}$ Jena University Hospital, Jena, Germany

In a collaboration project between the Integrated Biobank of Luxembourg (IBBL) and the Jena University Hospital (Germany), we evaluated (1) the fitness-for-purpose of a new blood collection tube, for demanding metabolomics analyses, and (2) the plasma pre-analytical quality control tools, which have been developed by IBBL and by Jena University Hospital to check for pre- and post-centrifugation delays.

The study was designed to compare the Barricor tube to the classical K2-EDTA tube, assessing three pre-centrifugation delays at room temperature $(1 \mathrm{~h}, 23 \mathrm{~h}, 53 \mathrm{~h})$, and three post-centrifugation delays, also at room temperature ( $0 \mathrm{~h}, 24 \mathrm{~h}$ and $48 \mathrm{~h})$. For all of these testing conditions, blood was collected from 5 volunteer donors and the obtained plasma samples were distributed in 8 aliquots to perform the QC tests, each time on a separate aliquot.

The Barricor blood collection tube contains a plastic separator which isolates the plasma from the other blood components upon centrifugation. According to the manufacturer, centrifugation can be done at $4000 \mathrm{~g}$ for $3 \mathrm{~min}$ only, and postcentrifugation shipment of pure plasma samples in the original tube is feasible. This saves time and resources.

To evaluate the fitness for-purpose of the Barricor tube for isolation of cfDNA, we spiked cfDNA mimics for the $1 \mathrm{~h}$ precentrifugation delay conditions, and then extracted cfDNA from isolated plasma and finally analysed the cfDNA content and profile of these samples.

Several quality control tests were performed on the collected samples: platelet count on the blood sample and platelet count (by CASY counting) on the isolated plasma samples after the imposed pre- and post-centrifugaton delays. The following QC tests were performed:

- sCD40L measurement by ELISA to assess the performance of this tool in the "diagnosis" of room temperature exposure of plasma or serum samples;

- IL-16 and IL-8 measurement to assess the performance of these tools in the "diagnosis" of pre-centrifugation of more than $24 \mathrm{~h}$ at RT;

- metabolites testing by LacaScore (lactate/ascorbate) and by LC-MS/MS at Jena University Hospital.

A comparative table with results obtained with these seven different tests will be presented as well as a summary table with the evaluation outcome.

\section{Biospecimen Research and Science - LUX}

\section{LBP-36 Biobanking of Biospecimens for the Epidemiology of Cardiovascular Risk Factors and Diseases in Regions of the Russian Federation Study (ESSE-RF)}

M. S. Pokrovskaya ${ }^{1}$, O. Sivakova ${ }^{1}$, A. N. Meshkov ${ }^{1}$, V. A. Metelskaya ${ }^{1}$, G. A. Muromtseva ${ }^{1}$, S. A. Shalnova ${ }^{1}$, S. V. Anisimov ${ }^{2}$, O. M. Drapkina ${ }^{1}$, S. A. Boytsov ${ }^{1}$

${ }^{1}$ National Research Center for Preventive Medicine of the Ministry of Healthcare of Russia, Moscow, Russian Federation, ${ }^{2}$ National BioService LLC, Saint-Petersburg, Russian Federation
Background: At the present moment the second phase of epidemiologic study ESSE-RF 2 is conducted in four Russian regions (Ryazan, Krasnodar, Omsk regions, republic of Karelia) and is coordinated by the National Medical Research Center for Preventive Medicine (NMRCPM). ESSE-RF2 targets investigation of epidemiology of cardiovascular diseases and their risk factors. Biobank of NMRCPM is able to store large amount of biospecimens. The aim of our study is organization of biospecimens biobanking from Russian regions.

Methods: Special schemes of interaction with local personnel were developed to set up correct and standardized procedures for blood draw, sample proceeding, labeling and shipping of biospecimens from the regions. Detailed instructions for every stage of work were prepared and training of all participating personnel was performed; all sample collection kits were prepared in our Biobank. Special informed consent forms that could be shipped together with biospecimens were designed. Representative sample of population was targeted for each region. Specially designed software for data entering has been developed by our Biobank.

Results: ESSE-RF2 study covered 4 regions, where biospecimens from $>6800$ subjects have been collected. All samples were shipped to and stored at NMRCPM Biobank. For each participant, 8 aliquots of serum $(2 \times 1.0 \mathrm{ml}+6 \times 0.5 \mathrm{ml}), 3$ of NaCitrate plasma (x $0.5 \mathrm{ml}), 3$ of EDTA plasma $(\mathrm{x} 0.5 \mathrm{ml}), 4 \mathrm{ml}$ EDTA whole blood and $2 \mathrm{ml}$ of EDTA RBC concentrate were stored. Shipping of frozen samples from regions to NMRCPM was conducted with strict temperature monitoring. All biospecimens and sample-associated data were collected within 3 months. Large amount of encoded detailed clinical and sociodemographic information from study participants was collected.

Conclusions: In Russian ESSE-RF2 epidemiological study, a centralized biobank provided key services related to personnel training, preparation means, sample collection, transportation, storage and data handling. To guarantee a quality of biospecimens and accompanying information in large epidemiological studies, effective organizational means are critical, covering work with participants in regions, shipping, temporary and long-term storage. Organizational schemes were tested and troubleshooted, not only supporting this important study but also providing a reliable foundation for the future studies led by NMRCPM.

\section{LBP-37 Develop and Validate PCR and ELISA Methods for Detecting Orthopoxvirus in Georgia}

\section{A. Gulbani}

\section{Laboratory of Ministry of Agriculture, Tbilisi, Georgia}

Background: The recent discovery (2013) of a new Orthopoxvirus (OPXV) in Georgia (country) demonstrates the need for poxvirus detection and diagnosis capacity in country. Human illness caused by this virus has implications for differential diagnosis of cutaneous lesion-producing zoonotic infections, principally anthrax. Simultaneously, animal infection may impact agricultural productivity and food safety. Because of the emergence of new pathogenic poxviruses, there is a great need for the development of PCR and ELISA methods for detecting poxvirus. Laboratory of Ministry of Agriculture (LMA) will develop laboratory capacity, recognition and reporting capacity, and human resources necessary to perform routine poxvirus surveillance in animals.

Methods: An optimized ELISA and PCR assay for the detection of orthopoxviruses are in the process of development by LMA researchers in collaboration with CDC. CDC provides training in assays for the detection and identification of pox- 
viruses, including standard PCR and quantitative PCR, and ELISA for detection of anti-OPXV IgG in serum.

Initial testing at LMA involved screening field collected swabs for the presence of OPXV according to published qPCR protocols ( $\mathrm{Li}$ et al. 2007). This is a generic assay designed to detect all OPXV species except Variola virus. In this way, will be recovered any additional new isolates or other species of OPXV that may be circulating in the region. Any positive samples will be further characterized to establish species identification and marked for genome sequencing.

Conclusion: New assays will be adopted and validated for detecting new OPXV variants in, rodents, domestic and wild animals found in Georgia.

Training and educational outreach will result in improved capacity for efficient identification and diagnosis of emerging OPXV, and will as well improve bio-surveillance capacity for OPXV in both human and animal populations.

The improved surveillance activities and understanding of OPXV disease burden in the agricultural sector will promote further research collaborations with local and international partners.

\section{LBP-38 Evaluating the Utility of Necropsied Marine Animal Tissues in Genomics}

\section{J. Ness, A. Moors, R. S. Pugh}

National Institute of Standards and Technology, Charleston, South Carolina, United States

The NIST Marine Environmental Specimen Bank is a useful tool for long term and retrospective studies relating to environmental health and the health of marine animals with respect to environmental contaminants. Focus on research relating to genetics, metabolomics and proteomics can support measured contaminant data by adding layers to our understanding of the effect contaminants have on animal health, life history, and the environment. The strict protocols established by NIST for the banking of animal tissues and fluid for environmental contaminants have also preserved sensitive biological molecules for many samples. Research materials banked at the Marine ESB are obtained from live and expired (through necropsy) marine animals. Many of these samples are from federal and state protected marine species and can be difficult for researchers to obtain due to permitting and sampling logistics, especially from live animals, and may be limited in the number and quantity of sample available. Expired animals however can provide larger quantities of tissues from internal organs that cannot be obtained from a live animal, which can also aid in understanding metabolism, genetic expression, and overall health. This research examines how RNA degradation and gene expression is altered in necropsied tissues stored at the Marine ESB for the National Marine Mammal Tissue Bank. It will explore the limitations of these tissues and provide a framework for researchers studying marine animals to determine if more accessible and abundant tissues from expired marine animals can be utilized for their studies.

\section{Biospecimen Research and Science - Quality - LBA}

\section{LBP-39 The Biobanking of Human Peripheral Blood Mononuclear Cells}

A. Zang, Y. Xu, C. Lei, S. Xue, D. Wang, L. Yao, X. He, H. Guo

Origincell Technology Group Co., Ltd., Shanghai, China
Human peripheral blood mononuclear cells (PBMCs) are required in multiple prospective and functional analyses in infectious diseases and clinical vaccine studies, as well as serving as precursors for potential immunotherapy development in translational medicine. Therefore, the biobanking of PBMCs is of growing importance. High quality of biobanked PBMCs positively contributes to their efficiently exploratory and future applications.

According to our findings here, age other than gender is a major autologous factor affecting PBMC cryopreservation. Upon thawing, PBMCs from people older than 60 years showed significantly less cell viability and cell recovery than those from younger ones. Besides determining the cell viability and recovery, we also developed multiple platforms to do phenotypic and functional evaluation of bio-banked PBMCs, which include the analysis of TBNK population, lymphocyte proliferation assay (LPA), T cell activation and subsequent effector cytokine secretion, NK expansion as well as dendritic cell differentiation. Consequently, here we showed a stable and durative good performance of PBMC cryopreservation strategy, with more than $90 \%$ of cell viability and $80 \%$ of cell recovery in average after their thawing, which provide strong basis for the construction of PBMC bio-bank.

\section{LBP-40 Three Methods for Leukocytes Separation and RNA Quality Control}

H. Zhang, L. Ruan, R. Gan

Shanghai Clinical Research Center, Shanghai, China

Background: Leukocytes functions in immune system as immune activation and downstream effector response, which indicated important roles in disease as well as the therapeutic candidate of tumors. This research explores three methods for leukocytes isolation from whole blood and RNA extraction for quality control.

Methods: Whole blood was collected into EDTA anticoagulation tubes. Lymphocyte separation medium (LSM) was used for leukocytes separation and we developed anther method of Red Blood Cell Lysis Buffer(RBC lysis) to isolate leukocytes. Also, optimized Lymphocyte separation medium method was implemented to purify leukocytes. After that, RNA was extracted from purified leukocytes and for quality control.

Results: RNA was extracted for quality control by Agilent 2100 , the mean value of RIN is above 8 by LSM method. Further, higher mean RIN value was obtained by optimized LSM method compared with LSM method. Also, RBC lysis method provided purified leukocytes, by which the mean value of RIN was above 8. Besides leukocytes, serum, platelet and red blood cells could also be obtained by optimized LSM and RBC lysis methods. Further, the RNA quality meet the downstream application from clinical samples by RBC methods.

Conclusion: Three methods is enough to satisfy leukocytes separation and RNA quality control. Among these, LSM method costs a lot of time. And optimized LSM method could reduce time cost. RBC lysis method is the simplest one, of which it take least time and processing procedure. Before leukocytes separation, serum, platelet and red blood cells could also be collected by optimized LSM method and RBC lysis method.

\section{LBP-41 The Proficiency Testing Program in National Biobank of Korea}

H. Kim, M. Lee, Y. Kim, Y. Kim, J. Jeon

National Institute of Health, Cheongju-si, Chungcheongbuk-do, Korea 
In 2013, the proficiency testing program was introduced to improve the accuracy and precision for quality management of biospecimens. From 2013 to 2015, we assessed the proficiency of DNA concentration and purity, RNA integrity, bacteria contamination in human genomic DNA and cell viability of 17 regional biobanks of the Korea Biobank Network (KBN) and clinical lab testing service organizations. In 2016, the proficiency testing (PT) was opened to all governmentlicensed biobanks across the country. The PT results were statistically evaluated according to ISO 13528:2005 Robust analysis or Q/Hampel method. In the first year of the PT program, in 2013, the conformance ratio of the participants was $95.5 \%$ (DNA concentration), $100 \%$ (DNA purity, RNA integrity and cell viability) and $86.4 \%$ (bacteria contamination in human genomic DNA). Since then, the conformance ratio of all PT schemes remained in a stable state more than $90 \%$ except for 2016. In addition, educational training courses for practical QC/QA were regularly conducted for various biobanks including KBN-member and non-member biobanks by the National Biobank of Korea, which contributed to improve the measurement capability and laboratory skills of participants.

\section{LBP-42 Determining Quality of BioBank Tissue Samples}

T. E. Tarling, S. Vercauteren, J. Terry, P. Lange, T. Soroski, J. Bush

Pathology and Laboratory Medicine, University of British Columbia, Vancouver, British Columbia, Canada

Background: In a hospital setting it is impossible to avoid delays between required pathological examination, sample collection and processing for research. Our goal is to set a protocol that is reflective of the real clinical work flow, while optimizing conditions for future tissue biobanking.

Methods: Tissue punches of tonsil samples retrieved from consenting patients were immediately snap frozen in liquid nitrogen in the operating room (OR). Remaining tissue was divided, with half being stored in preservation buffer and half being stored on saline-soaked gauze at room temperature for 30 minutes to simulate the anticipated time to receive tissue from the OR in the biobank. Punches were taken from each condition (buffer and saline) after 30 minutes and stored at $-80 \mathrm{C}$. All remaining tissue was transferred to $4 \mathrm{C}$. Over the course of 7 days punches were taken from tissue in each condition at 2, 24, 48, 72, and 168 hours and were stored at $-80 \mathrm{C}$ until analysis.

The quality of nucleic acids and proteins from the punch samples were analyzed quantitatively. Gross and histologic assessment was used to compare frozen tissue versus tissue submerged in a preservation buffer.

Results: Preliminary results show that DNA concentration from tissue stored on saline-soaked gauze at $4 \mathrm{C}$ over a 168 hours is comparable to DNA concentration from tissue that was immediately snap frozen. However, when tissue was stored in buffer at 4C, DNA concentration was stable up to 72 hours but declined significantly between the 72-168 time interval $(\mathrm{P}<0.01)$. No significant change in DNA purity (A260/A280 and A260/A230 ratios) was found between the different storage conditions and DNA degradation mildly increased over time under both storage conditions. When tissue is stored in saline at $4 \mathrm{C}$, the RNA concentration is stable up until 72 hours. When stored in preservation buffer at $4 \mathrm{C}$, the RNA concentrations are more variable and decrease after 48 hours.

\section{Biospecimen Research and Science - Quality - LUX}

\section{LBP-43 Stability of Total and Free Prostate Specific Antigen After Ten Years Storage}

\author{
J. Kinkorova ${ }^{1}$, V. Simanek ${ }^{2}$, O. Topolcan ${ }^{1}$, M. Karlikova ${ }^{1}$, \\ O. Dolejsova ${ }^{3}$, R. Kucera ${ }^{1}$
}

${ }^{1}$ Department of Immunochemistry, University Hospital Pilsen, Pilsen, Czechia, ${ }^{2}$ University Hospital Pilsen, Pilsen, Czechia, ${ }^{3}$ Department of Urology, University Hospital Pilsen, Pilsen, Czechia

Background: PSA is a serine protease composed of 240 amino acids in a single polypeptide chain and is a routine parameter in prostate cancer diagnostics. Retrospective research requires an accurate knowledge of the stability of the biomarker molecules. The aim of our study was to test the long term stability of tPSA and fPSA after ten years storage at $-80^{\circ} \mathrm{C}$.

Methods: We analyzed two aliquots from 50 serum samples. Serum was separated within 3 hours of blood collection. Serum samples were immediately aliquoted and processed or frozen at $-80^{\circ} \mathrm{C}$. The first aliquot was assayed in routine testing in 2006. The second was thawed for further testing after ten years storage at $-80^{\circ} \mathrm{C}$. We compared 50 results of tPSA, 20 results of tPSA and 20 calculated results of the fPSA/ tPSA ratio. Serum tPSA and fPSA levels were assayed using chemiluminescent kits Access Hybritech PSA and free PSA (Beckman Coulter, USA). All statistical analyses were performed by SAS 9.3 software.

Results: The mean decrease in fPSA after 10 years stocking was $4.5 \%$. fPSA increased $9.4 \%$ on average and fPSA/tPSA ratio increased $18.1 \%$ on average. On clinical evaluation three samples dropped to the lower category of malignancy.

Conclusion: Stability of tPSA levels is sufficient after 10 years stocking at $-80^{\circ} \mathrm{C}$. fPSA is less stable and its using after ten years is limited. The calculation of the fPSA/tPSA ratio is not recommended due to the alteration towards false negative results.

\section{LBP-44 Standardized DNA and RNA Sample Quality Control}

E. Viering

Marketing, Agilent Technologies R\&D and Marketing GmbH \& Co KG, Waldbronn, Germany

Many factors have an influence on nucleic acid quality, such as sample source, handling, extraction method and storage condition. The responsibility of the biobanks to collect, store and ship samples makes it essential to determine sample quality at the point of receipt and release. Sample quality includes concentration and integrity which are both important parameters to ensure that nucleic acid samples are fit for purpose. Nucleic acid quality can be assessed using conventional gel or automated electrophoresis systems.

The DNA Integrity Number (DIN) has been established for genomic DNA (gDNA) qualification with automated electrophoresis systems. It provides an assessment of gDNA sample quality by assigning a numerical score from 1 to 10 . A high DIN indicates intact gDNA, and a low DIN degraded gDNA. The DIN enables comparison of samples and allows defining a DIN quality threshold for specific types of samples or preparation. This poster shows examples of DNA sample patterns and cor- 
relating DIN across a wide quality range for DNA originating from blood, fresh frozen tissue and formalin fixed paraffin embedded (FFPE) material.

The RNA integrity number equivalent (RINe) delivers an objective assessment of total RNA degradation for samples from eukaryotic or prokaryotic origin. The RIN is independent of sample concentration and analyst and allows unbiased confirmation of RNA sample quality. RNA samples extracted from FFPE tissue are typically highly degraded. Many tailored FFPE RNA library protocols use an additional quality metric DV200 to define the optimal RNA input amount for successful NGS library preparation. The DV200 represents the percentage of RNA fragments above 200 nucleotides. This poster exhibits sample patterns and corresponding quality scores of intact and degraded RNA including FFPE RNA samples.

\section{LBP-45 OPTIMARK Project: Preliminary Results on Antigenicity and Integrity in Non-Tumor Tissue Samples}

C. Villena ${ }^{1,2}$, I. Almenara ${ }^{2,3}$, M. J. Artiga ${ }^{2,3}$, O. Bahamonde ${ }^{2,4}$, R. Bermudo ${ }^{2,5}$, E. Castro ${ }^{2,6}$, R. De la Puente ${ }^{2,7}$, T. Escámez ${ }^{2,8}$, M. Esteva-Socias 1,2, M. Fraga ${ }^{2,9}$, L. Jauregui-Mosquera ${ }^{2,10}$, M. Martin-Arruti ${ }^{6}$, M. Morente ${ }^{2,3}$, L. Peiró-Chova ${ }^{2,4}$, D. G. Pons ${ }^{1,2}$, J. D. Rejón ${ }^{2,7}$, M. Ruiz-Miró ${ }^{2,11}$, P. Vieiro ${ }^{2,9}$, S. Zazo ${ }^{2,12}$, V. Villar ${ }^{2,10}$, A. Y. Rábano, ${ }^{2,13}$, R\&D working group of Spanish Biobank Platform, ISCIII, Madrid, Spain

${ }^{1}$ Pulmonary Biobank Consortium, CIBER of Respiratory Diseases (CIBERES) - ISCIII (Madrid), Instituto de Investigación Sanitaria de Baleares (IdISBA) - Hospital Universitario Son Espases, Mallorca, ${ }^{2}$ Spanish Biobank Platform, ISCIII, ${ }^{3}$ CNIO Biobank, Spanish National Cancer Research Center (CNIO), Madrid, ${ }^{4}$ INCLIVA Biobank, Valencia, ${ }^{5}$ HCB-iIDIBAPS Biobank, Instituto de Investigaciones Biomédicas August Pi i Sunyer (IDIBAPS), Barcelona, ${ }^{6}$ Basque Biobank; The Basque Foundation for Health Innovation and Research (BIOEF), Bilbao, ${ }^{7}$ Andalusian Public Health System Biobank, Granada, ${ }^{8}$ IMIB Arrixaca Biobank, Murcia, ${ }^{9}$ Biobank of Research Institute of Santiago de Compostela, Santiago de Compostela, ${ }^{10}$ University of Navarra's Biobank IdiSNA, Pamplona, ${ }^{11}$ IRBLleida Biobank, Instituto de Investigaciones Biomédica de Lleida-Fundación Dr. Pifarre, Lérida, ${ }^{12}$ Fundación Jiménez Díaz Biobank, Madrid, ${ }^{13}$ Departamento Neuropatología, Banco de Tejidos Cien, Fundación Centro Investigación Enfermedades Neurológicas (CIEN), Madrid

Background: The development of many potential disease biomarkers on clinical assistance is limited by the sample quality, affected essentially by procurement, processing and storage conditions. So, many efforts had been made to standardize biological material preservation, although emerging biospecimen science is focusing on study the impact of critical factors over the samples that could affect on the accurate biomarker analysis. In that context, OPTIMARK project, a multicenter initiative carried out by 12 centers with the collaboration of R\&D working group of Spanish National Biobank Network, aims to select and validate the essential pre-analytical factors relevant on tissue samples through an algorithm which consider SPREC and BRISQ information and some analytical testing with high predictive value.

Methods: The first phase focused on identifying analytical tools to validate the quality of tissue samples, based on its integrity and antigenicity, in order to evaluate the effect of long term storage. A total of 374 retrospective non-tumor tissue samples (colon, brain, lung, breast, stomach and endometrium) preserved from less than a 1 year old to more than 20 years were tested. In order to evaluate quality of antigenicity, different cellular markers of ubiquitous distribution among tissues were selected, according to Human Protein Atlas database on the formalin-fixed paraffin-embedded (FFPE) non tumor tissue samples. At the same time, RNA integrity number (RIN) was also evaluated for most paired frozen samples using Agilent 2100 Bioanalyzer.

Results: Ki-67 protein was identified as a potential quality biomarker for non-proliferative tissues when long term storage effect was evaluated. However, vimentin and CD31 proteins showed no differences on immunostaining quality between groups. Additional markers recently tested in lung tissue samples that showed a slight correlation between staining intensity and sample age were TTF-1, BCL-2 and beta-catenin. Except on gastric samples, optimal RIN values were obtained, although with high variability, maximum in brain samples. No correlation was observed between the RIN and long term storage. Only in colon, breast and endometrium samples was a slightly reduction of RIN values among time.

Conclusions: Correlations have been found on loss of antigenicity according to sample age depending on the marker used. However, the effect of long term storage on RNA integrity of frozen samples seems to be affected by other factors.

\section{LBP-46 Long-Term Stability of Tumor Markers in Human Sera}

\section{Karlikova, R. Kucera, O. Topolcan, J. Kinkorova}

Department of Immunochemistry, University Hospital and Faculty of Medicine in Pilsen, Czech Republic, Pilsen, Czechia

Background: Serum tumor markers are biomarkers used on a routine basis in clinical decision making in oncology. Retrospective research requires an accurate knowledge of the stability of the biomaker molecules.

Aim of study: To identify the changes in serum tumor markers levels after six to ten years of storage at $-80^{\circ} \mathrm{C}$. Selected tumor markers were: carcinoembryonic antigen (CEA), cancer antigen 19-9 (CA 19-9), tissue polypeptide specific antigen (TPS), insuline-like growth factor (IGF-1) and thymidine kinase (TK).

Methods: Tumor markers were first assessed in serum samples after sample withdrawal in 2006 and again in 2016, after a storage in $-80^{\circ} \mathrm{C}$. The same methods were used: chemiluminiscence (CEA, CA 19-9) and immunoradioassay (IGF-1, TPS). Results were compared using Spearman correlation.

Results: IGF-1 levels decreased to $94.7 \%$ of original values (mean), the difference was statistically significant. CA 19-9 values increased to $100.8 \%$ of original values, the increase was statistically insignificant. TK TPS and CEA values increased to 112,114 and $125 \%$ of original values, respectively; the increase was not statistically significant. There was a strong correlation between results for all markers.

Conclusion: We did not noticed any significant level decrease of studied markers which implies that there was no significant molecule degradation. Serum samples stored at $-800 \mathrm{C}$ for 10 years can be used for clinical studies. 


\section{Ethical, Legal, and Societal Issues - LBA}

\section{LBP-47 Tackling Compliance Challenges for Use of Biological Assets in Drug Discovery}

S. Quirin

GlaxoSmithKline, Collegeville, Pennsylvania, United States

Biological assets play a vital role in drug discovery and development. Maintaining compliance with third party agreement and licensing terms for biological materials sourced from academic and government institutions, as well as from wellestablished repositories, can prove to be a challenge for large international pharmaceutical companies. This challenge is magnified due to increasing third party interactions with external collaborators and contract research organizations. ABS compliance for the Nagoya Protocol adds an additional layer of governance required for biological research materials used in R\&D. This poster discusses these challenges and the approach taken by GlaxoSmithKline to centralize license and agreement acquisition, enforce data registration, and implement tracking measures to improve and strengthen the compliance framework surrounding our biological assets.

\section{LBP-48 Implementing Electronic Consent in an Academic Biorepository}

T. B. Daughrity, M. K. Washington, K. R. Wiles, M. Manrao

Pathology, Microbiology, and Immunology, Vanderbilt University Medical Center, Nashville, Tennessee, United States

The Cooperative Human Tissue Network at Vanderbilt University Medical Center (CHTN-VUMC) is a federally funded academic biorepository that is dedicated to providing high quality biospecimens and services to the research community. For 15 years, CHTN-VUMC has been obtaining broad consent from patients to collect samples for a wide variety of research studies. Due to the complexity and regulatory requirements, the consent process is inherently time-consuming and a multi-step process, which utilizes staff time and money. CHTN-VUMC routinely implements process improvements and the adoption of Lean Six Sigma (LSS) principles within our biorepository, which has driven the decision to implement digital consent documentation. Wastes that could be reduced were defined as inventory, motion, defects, and extra processing. According to CHTN-VUMC's error-reporting software, $25 \%$ of consenting errors in 2017 were categorized as major or critical. In an effort to address these wastes, the current paper process was evaluated against the digital consent proposal through SIPOC and Fishbone diagrams to identify all relevant elements of the process improvement before a commitment was made to implement digital workflows.

Through the use of LSS diagrams, the root cause of waste was determined to be the sizable number of steps in the consenting process. CHTN-VUMC's goal for implementing electronic consent (eConsent) is to save around $\$ 2600$ that was previously spent on printing costs and staff time, as well as reduce the percentage of major or critical consenting errors to less than $15 \%$. The launch of CHTN-VUMC's eConsent will be through Research Electronic Data Capture (REDCap), VUMC's secure web-based application for surveys and databases. REDCap will allow patients to be consented using an iPad to capture a "wet signature" using a finger or stylus rather than by traditional paper consent. Following LSS principles has allowed CHTN-VUMC to operate efficiently using limited resources.
Putting these ideals into practice via eConsent will allow the lab to significantly reduce waste and allow for more focus to be on providing the highest quality biospecimen to researchers.

\section{LBP-49 Parental Attitudes and Willingness to Donate Children's Biospecimens for Congenital Heart Disease Research}

\section{S. Liu}

Department of Biobank, Shanghai Children's Medical Center, Shanghai, China

Background: High-quality biospecimens from consenting patients are crucial for congenital heart disease (CHD) research. This study assessed attitudes and willingness of parents of toddlers with CHD regarding donating biospecimens for CHD research, and identified factors associated with positive attitudes toward biospecimen donation.

Methods: A cross-sectional survey was conducted among parents of children with CHD that attended the Shanghai Children's Medical Center cardiothoracic surgery inpatient department from March 1, 2016 to December 31, 2016. The anonymous questionnaire covered parental demographic data; willingness and motivation regarding donating their children's biospecimens; and ethical and legal considerations concerning their children's future willingness to donate biospecimens.

Results: The sample comprised 550 parents; 508 completed the questionnaire (response rate 92.4\%). In total, 69.1\% $(\mathrm{n}=351)$ participants were willing to donate biospecimens for medical research. Multivariate analysis indicated that education level (odds ratio [OR] $0.401 ; 95 \%$ confidence interval [CI]: 0.202-0.796, $\mathrm{p}=0.009$ ), and children's history of hospitalization (OR 0.638 ; 95\% CI: $0.433-0.939, \mathrm{p}=0.023$ ) were positively associated with willingness to donate biospecimens. The most common motivation for donating biospecimens was the potential benefit to other children with CHD $(81.2 \%, \mathrm{n}=285)$. In addition, $86.0 \%(\mathrm{n}=302)$ of participants expected to be informed of the research results from their children's donated biospecimens.

Conclusions: Nearly $70 \%$ of parents were willing to donate their children's biospecimens for CHD research. Parents' education level and children's history of hospitalization influence willingness to donate biospecimens. A majority of parents expect to receive the research results for children's biospecimens. Keywords: Congenital heart disease, biospecimen donation, parents' willingness

\section{LBP-50 The Re-Consenting Hurdles of a Pediatric Biorepository}

\section{W. Marsh, L. Hlivka, D. Carpentieri}

Pathology, Phoenix Children Hospital, Phoenix, Arizona, United States

Background: Consenting at the age of majority is an administrative hurdle for many biorepositories, especially those in a purely pediatric realm. Many pediatric participants are seen up to the age of 18 , but once the patient is over that age, they find new primary care doctors. This can lead to its own challenges such as keeping an updated mailing address, or contact information when there are large gaps in which the patient is seen at the hospital. While other avenues exist for retaining their donated material, such as waivers, those typically come with heavy costs to what the tissue can be used for, and ultimately is not preferred. 
Methods: Biorepository staff identified participants who have turn 18 years of age in order to attempt to obtain an updated consent. Once these participants were verified by Biorepository coordinator as alive, and neurologically capable, the subjects most current contact information and mailing address was obtained through the hospital electronic records. Consent packets were created containing a study introduction letter, consents with required fields highlighted, and all return mailing supplies. These were then mailed out to participants via certified mail. The coordinator then attempted to contact participants within 10 days of delivery receipt to address any questions about the study, or any study documentation.

Results: Of the 77 participants who turned 18 years old, 51 consents were sent out. 22 consents were not sent either due to the lack of samples, or due to concerns over neurological impairment. Of the 51 consents sent out, 30 were successfully delivered, and 21 were unsuccessful (18 due to a change in participants address and 3 that were unknown). Of the 30 that were delivered, 12 were successfully returned; however, only nine of the 12 were completed correctly. The three incorrectly completed consents were either signed by the parents, not the participant, or were missing time and dates.

Conclusions: Ensuring up-to-date information and creating consent packages takes valuable time, and has shown a limited return on investment (9/77). While waivers can be obtained, or samples kept under IRB stipulations (I.E. fully autotomized), these are sub-optimal in regards to an actual consent. A protective and realistic guideline is needed.

\section{LBP-51 Reflexivity and Ethics for ABS of the CBD: Empirical Analysis by Network of Scientific Articles with NLP}

R. Kohsaka ${ }^{1}$, H. Matsuoka ${ }^{2}$, A. Tashiro ${ }^{1}$, Y. Uchiyama ${ }^{1}$

${ }^{1}$ Graduate School of Environmental Studies, Tohoku University, Sendai, Miyagi, Japan, ${ }^{2}$ Kanazawa University, Kanazawa, Japan

ABS has global challenge for decades, particularly since the birth of CBD. Analysis have largely been conducted separately at various disciplines (ecology, biology, pharmaceutics, law, ethics) and the interlinkages, despite their importance, remain unexplored.

We have selected 176 papers from the database of the PubMed and the Web of Science by using the keyword "Access and Benefit Sharing." We have focused on disciplines, regions and relationships between the academic papers that discuss ABS issues. The relationships of the academic discourse can be analyzed by focusing on the citations between the papers. We scanned all papers in the reference lists of the 176 selected papers and detected cited papers in the selected papers.

As first step, we identified disciplines where ABS related publications are found and referred to. We also identified regional specific trends, where focus in Africa was on bioprospecting while the discourse in Asia was linked to culture. For example, frequently mentioned terms in the research of Asia include taro, horticulture, ornamental, and cultivar. In the research of Africa, the terms that include bioprospecting, medicine, industry are relatively frequently mentioned. On the other hand, in the research of Europe, the terms that include conservative, achievement, leadership, performance and objective are frequently mentioned and the related research topics are discussed. As the second step, we grouped the references networks and found that the discourses are fragmented into three groups and others.

These results, limited to the scope of academic papers, suggest that it is necessary to share the multi-and trans- disciplinary approaches. There are clusters of the papers from the similar disciplines. The journals, including this journal, Biopreservation and Biobanking, are identified as a hub for different discipline and play a role as catalysis. Because the issues of ABS need to be addressed by the multi-and transdisciplinary approaches, the catalyst that can connect the approaches from different disciplines is indispensable, and the research focusing on the science policy interface and participatory approached of various stakeholders need to be implemented with international collaboration including academia, industry and local communities.

\section{Hot Topics - LBA}

\section{LBP-52 A Brief Introduction of ABC2017}

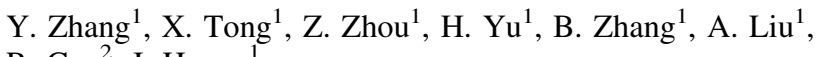
R. Gan ${ }^{2}$, J. Huang ${ }^{1}$

${ }^{1}$ Anatomy and Neurobiology, Basic Medical School, Central South University, Changsha, Hunan, China, ${ }^{2}$ Shanghai Clinical Research Center, Shanghai, China

The Annual Biobank China (ABC) Conference is as an interactive and innovative conference experience which promotes the development of the Chinese biobanking system and offers a great platform to provide further education to Chinese biobankers. Six ABC conferences have established a global network of professionals, providing the opportunity and momentum for major progress in the development of biobanking and related medical research.

The Annual Biobank China 2017 \& International Symposium on Precision Medicine, (ABC2017), is the latest ABC meeting. ABC2017 was successfully held in Changsha, China on December $1^{\text {st }}-2^{\text {nd }}, 2017$, organized by the Central South University (CSU), with invaluable assistance from the Shanghai Clinical Research Center (SCRC) and the Shanghai Engineering Research Center of Biobank (SERCB).

$\mathrm{ABC} 2017$ succeeded in inviting more than 100 leading biobanking researchers as speakers. It also attracted more than 1000 participants from 8 countries who were mainly biobankers, academics, other top scientists, manufacturers, and policy makers. Comprising 5 plenary symposia, 5 parallel sessions, 2 seminars and 2 special programs, ABC2017 promoted consensus on quality control and standardization system, ethical and regulation issues, sustainable development strategy and application of big data, automation and artificial intelligence(AI) in biobanking construction and management. ABC2017 also promoted a broad discussion on precision medicine and next-generation biobank construction between biobankers operating under different laws and ethical regulations, different networks, different business models, variable development status and different governmental strategies. During this meeting, the Hunan Industry Technology Innovation Strategic Alliance was founded, an AI specimen storage system from Genepoint Biological Technology (Shanghai) was released. In particular, ABC2017 was the first ABC meeting which held a photographic exhibition, showing the history of biobanking in China and interesting stories from biobankers across the world.

Sincerely, we would like to thank Prof. Jim Vaught and other chairpersons, professionals, volunteers for their efforts during this meeting, which not only contributed to the success of this inter-disciplinary, multi-field conference, but also will promote worldwide cooperation among country-based health and technology programs.

This work was funded by the National Key Research and Development Program of China (2016YFC1201800). 


\section{LBP-53 Pathogen Asset Control System (PACS) Integration with Radiofrequency Identification (RFID) Technology at NCDC of Georgia}

\author{
S. Chubinidze, M. Grdzelidze, G. Mgeladze, S. Tsanava
}

Biosafety and EDP, National Center for Disease Control and Public Health, Tbilisi, Georgia

The National Repository of Bacteria and Viruses (NRB\&V) is a part of National Center for Disease Control and Public Heath(NCDC) of Georgia. There are kept collection of Especially Dangerous Pathogens - obtained from humans and environment, other pathogenic and potentially pathogenic microorganisms that are of a scientific or practical value and reference strains; The Pathogen Asset Control System (PACS) is implemented and successfully used for biological agent stocks accounting and control, that is a secure, comprehensive information system developed under Cooperative Biological Engagement Program that is implemented by the Defense Thread Reduction Agency (DTRA) to track biological materials. In order to increase Biosafety/Biosecurity capacity at biological laboratories, a pilot project was made to integrate PACS radiofrequency identification (RFID) technology. The goal of the integration was not only to allow enhanced security of storage but also to simplify tracking and improve effectiveness of laboratory operations. Pilot Project was implemented in the NRB\&V located at the Lugar Center. PACS-RFID System included following components: Standalone workstation with PACS database and application; Barcode printer; Barcode scanner; RFID reader and desktop antenna; RFID plate reader; RFID-enabled -80C freezer (manufacturer - Terso Solutions); RFID printer; Plastic vials with embedded RFID tags; Variety of RFID tags to be used with existing vials. Each component was used for specific purposes. Methods included: New material registration, Material transfer, Material subculturing, Material aliquoting, Material destruction, Inventory Audit, Freezer Operations. Pilot Project showed that not all RFID technology enabled equipment provides benefits of the same level, some equipment tested during the project was not deemed reliable (e.g. RFID-enabled non-sterile vials). Project participants identified that RFID technology increased security of biological materials storage and tracking but to a limited level (e.g. boxlevel tracking by Terso Freezer), generally increased efficiency of operations (e.g. fast inventory), as well as improved accuracy. It was identified that RFID technology increased convenience and speed of operations with PACS, especially inventory audit, as well as enhanced general accuracy. At the same time, it provided limited benefits to the security as the technology still requires further improvement - e.g. vial-level tracking by Terso Freezer.

\section{LBP-54 ACMG Incidental Findings at the CLIA-Certified Colorado Center for Personalized Medicine Biobank}

\section{S. J. Wicks, K. C. Barnes, K. R. Crooks}

Colorado Center for Personalized Medicine (CCPM), University of Colorado, Anschutz Medical Campus, Aurora, Colorado, United States

Background: The Colorado Center for Personalized Medicine (CCPM) has been established to uncover advancements in genomics that can improve diagnosis and treatment of disease. To hasten discoveries in personalized medicine, the CCPM has established a CLIA-certified human specimen Biobank. Ac- companying biospecimen collection, we harness patient health information to support a broad range of research, operational, and clinical quality improvement agendas. To date, we have enrolled over 53,000 adult subjects from across the University of Colorado Health (UCHealth) system into the Biobank and collected blood samples from over 23,000 subjects.

Methods: DNA extracted from patient samples undergoes microarray genotyping using a custom Infinium Multi-Ethnic Global Array SNP chip (MEGA, Illumina, Inc.). Among the $\sim 2.1$ million genetic variants on the microarray are several thousand with potential clinical relevance. The CCPM Biobank follows the current American College of Medical Genetics and Genomics (ACMG) recommendations for reporting of incidental genetic findings (PMID 27854360). Here we present information on the incidence and characteristics of reportable incidental findings from the first 1,500+ genotyped patient samples.

Results: MEGA content includes variants classified in ClinVar as "pathogenic" or "likely pathogenic" in each of the 59 ACMG genes. The content varies by gene, with 56 genes having between 1-80 potentially reportable variants (average 17). The remaining 3 genes, BRCA1, BRCA2, and COL $3 A 1$, have greater representation on the microarray, with 225,250 , and 258 pathogenic/likely pathogenic variants, respectively. Samples with identified variants have then undergone orthogonal confirmation by Sanger sequencing. After consenting to the return of these results, participants will be informed of the results by genetic counselor or clinical geneticist. The average incidental finding rate is approximately $1 \%$ to date. As expected given the microarray content and estimated allele frequencies, BRCA1 and BRCA2 are relatively over-represented and together account for $>50 \%$ of the incidental findings. The remainder is distributed among several genes, including GLA, DSG2, LMNA, MYBPC3, and others.

Conclusions: The MEGA microarray combined with Sanger confirmation provides a powerful tool for identifying pathogenic or likely pathogenic reportable genetic variants in a general population Biobank setting.

\section{LBP-55 Biobanks Managing Specimen and Annotations Associated with Patient Derived Xenografts (PDX)}

\section{H. Dowst, S. Hilsenbeck, M. Lewis}

\section{Baylor College of Medicine, Houston, Texas, United States}

Statement of Problem: Patient Derived Xenografts (PDX) are created by transplanting patient tissue specimen in mice. PDX models are used as patient "avatars" to enable translational research on living patient tissues. Baylor College of Medicine (BCM) established a PDX Core to optimize techniques and procedures for PDX model development. The PDX Core has been successful in establishing over 100 stable PDX models and plans to ramp up development. In 2016, the Core realized their current data management methods were inadequate to manage generations of PDX models, associated stored specimen, and clinical data sets. The Dan L. Duncan Cancer Center biobank at BCM was approached by the PDX Core to assist in developing a process and pipeline for managing the PDX Model data.

Proposed Solution: PDX Model tracking has many similarities to human tissue biobanking and is a natural extension of existing BCM biobank inventory and electronic data capture tool, Acquire. Acquire is an eForms engine for annotating participants registered in the BCM biobanks. In phase I, the 
inventory tracking system was extended to include the PDX module inventory, linking the model to the patient sample. Each transplant generation is logged as derivatives of the parent specimen which is distributed to the PDX Core. Through the use of roles and privileges in the application, Acquire separates the patient from the PDX model while still maintaining a data base link invisible to the PDX core. Using the eForms engine and dynamic reporting tool, rich clinical annotations including de-identified patient information such as pathology, molecular characterization, and treatment responses is being collected. Phase II, begun in late 2017, adds a PDX Visualization Portal combing the patient and PDX data. The portal displays data collected in collection-wide views and individual models in detail.

Conclusions: Since PDX Models have many of the same characteristics and tracking needs as biobanking, it is a natural extension of the biobanking systems to support PDX model development. Additional reporting and data visualization support is needed to link PDX models and the patient medical history. A community consensus on necessary tools is beginning to develop within the PDX research communities. BCM has both learned from and contributed to several national PDX projects such as the BCRF funded cBioPortal PDX site and the NCI funded PDXnet in establishing ontologies, data models, and tracking PDX models.

\section{Human Specimen Repositories - LBA}

LBP-56 MW CHTN Researcher Requested Tissue Sample Sizes (Weight) Distributed, 2015-2017: Supporting Data for Biorepository Research Tissue Sample Weights for Storage

R. Mandt ${ }^{1,2}$, D. G. Nohle ${ }^{1,2}$, M. E. Couce ${ }^{1,3}$, A. V. Parwani ${ }^{1,2}$, L. W. Ayers ${ }^{1,2}$

${ }^{1}$ CHTN-MW Division, Columbus, Ohio, United States, ${ }^{2}$ Pathology, The Ohio State University, Columbus, Ohio, United States, ${ }^{3}$ Pathology, Case Western Reserve University, Cleveland, Ohio, United States

Background: Midwestern Division (MW), Cooperative Human Tissue Network (CHTN) is a National Cancer Institute (NCI/NIH) funded program to provide quality research biospecimens to qualified investigators. Consented tissue samples are procured according to researcher specifications for size (weight) and preservation with the exception of those samples in significant demand and limited supply may have negotiated weights.

Methods: Shipped tissue sample weights were mined in CHTN software (Research Tissue Procurement Information System) for years 2015, 2016 and 2017. Samples by year and anatomic site for only primary malignant tissue samples with weight recorded (vs. size dimensions or liquid volumes) were included. Statistics included number of samples for each year and anatomic site as well as minimum, maximum, average, standard deviation and standard error. Anatomic sites with too few examples were excluded. "Stock price" type graphs were constructed to show an average as "volume" with both full weight ranges and range that accommodated $80 \%$ of samples.

Results: The number of samples (N) shipped is 2,119 in $2015,1,464$ in 2016 and 1,558 in 2017. The number of tissue samples and average weight by anatomic site included flat, rising, and falling trends; each anatomic site has its own pattern. Tissue weights fell into four groups; 10 and 90 percentile boundaries were calculated for each. Smallest average research tissue weights for middle $80 \%$ were recorded for prostate and oropharynx $(140 \mathrm{mg})$. Second weight group included tonsil, thyroid, breast, oral cavity, larynx, pancreas, salivary gland, skin, tongue, lung and parotid $(265 \mathrm{mg})$. Third group included stomach, cervix, colon, esophagus, endometrium, bone, brain, bladder, small bowel, uterus, liver, kidney lymph node, adrenal and ovary $(513 \mathrm{mg}$ ). The fourth and largest weight group included soft tissue and spleen at average distributed weight of $1201 \mathrm{mg}$.

Conclusions: Since tissue weights are not usually included in recommendations for frozen tissues stored in biorepositories, we offer this data as a practical guide to researcher acceptable tissue weights for selected sites for stored frozen tissue samples based on CHTN researcher request history.

\section{LBP-57 Insights into the Development and Clinical Outcomes of Atrio Ventricular Septal Defect- A Multicenter Tissue Banking Approach}

L. Wadhwa ${ }^{1}$, H. Lester ${ }^{2}$, B. Williams ${ }^{3}$, C. D. Fraser ${ }^{1}$, B. DeCampli ${ }^{4}$, I. Moslowitz ${ }^{5}$, C. Maslen ${ }^{6}$, J. O'Brien ${ }^{7}$, D. Bittel ${ }^{7}$, L. D' Allessandro', J. Marshall ${ }^{7}$, P. Gruber ${ }^{2}$

${ }^{1}$ Texas Children's Hospital, Houston, Texas, USA, ${ }^{2}$ University of Southern California, Los Angeles, California, ${ }^{3}$ Sick Kids Hospital, Toronto, Canada, ${ }^{4}$ Arnold Palmer Hospital for Children, Orlando, Florida, ${ }^{5}$ University of California, California, ${ }^{6}$ Oregon Health \& Science University, Portland, Oregon, ${ }^{7}$ Children's Mercy Hospital and Clinics, Kansas City

Congenital heart disease (CHD) is associated with life-long morbidities, premature mortality, and high health care costs. Atrio ventricular canal defects (AVSD) account for about 5 percent of all congenital heart disease, and are most common in infants with Down syndrome and is associated with variable outcomes. The genetic etiology of atrioventricular septal defect remains unknown in $40 \%$ cases. To overcome the lack of suitable number of samples sets, a multicenter approach was utilized. This study is the first project of an international initiative called CHSS Registry of Biobanks, created to engage in genomic research and foster collaborations among networks of biobanks of congenital heart diseases. A group of 7 centers in the US and Canada currently participate by sharing data and samples. In this project, we defined the genetic landscape of AVSD using next-generation whole exome sequencing in a cohort of 230 unrelated AVSD probands, syndromic and non-syndromic, collected from biobanks of four different collaborative groups. Variant filtering analysis revealed a complex genetic landscape with 78,644 rare variants identified in the cohort that are being further analyzed. Though the analysis is still in process, the Registry has enabled a collaborative project that received Federal grant support and has now completed sequencing. It establishes a new paradigm for the broad sharing of genomic resources and information in congenital heart disease.

\section{LBP-58 Biospecimen Collection and Management in a Large International Intervention Study}

J. F. Kemp, J. E. Westcott, K. Hambidge, N. F. Krebs, W. Preconception Nutrition Trial Group

\section{University of Colorado Denver, Aurora, Colorado, United} States

Background: Our goal is to improve maternal and child health in limited resource countries with low cost and sustainable interventions that build local infrastructure and capacity. 
The Women First Preconception Nutrition Trial is a randomized control trial in four Global Network sites located in India, Guatemala, Pakistan and the Democratic Republic of the Congo (DRC). The study aims to evaluate the effects of a maternal comprehensive nutrition supplement commenced at either $\geq 3$ months prior to conception, in the first trimester of pregnancy or not at all.

Methods: Biospecimens were collected longitudinally from a subset of mothers and their infants at each site. Depending on the sample type, specimens were collected for 50 or 100 mother infant pairs per arm. Biospecimen types include whole blood on blood spot cards, serum, plasma, red blood cells, urine, stool, buccal swabs, birth tissues, cord blood serum and breast milk. We modified standard operating procedures for biospecimen collection and transport to adapt to the capabilities of each site. Most supplies required for collections were shipped from UCD, whereas the sites provided some supplies they procured locally. Pre-printed barcoded cryogenic labels were supplied by UCD for all biospecimens. We utilize the TrackVia (Denver, CO, USA) platform for biospecimen tracking.

Results: We encountered various challenges to obtaining optimally handled biospecimens. The high humidity and ambient temperature in the DRC made drying blood spot cards prior to storage difficult. Shipping challenges included a regulation in Pakistan prohibiting the shipment of ice packs on airplanes, difficulty procuring temperature monitor devices in Pakistan and delays in shipments. Upon arrival at UCD, barcodes for all biospecimens were scanned and the samples transferred to storage locations. To date, 43,000 biospecimens have been received. Shipments for the primary study spanned several years and are now complete.

Conclusions: Thousands of biospecimens with different collection and handling requirements were successfully collected, stored and transported to the biorepository at UCD. We met our goal of obtaining longitudinal collections at all four sites. The capacity and knowledge gained about biospecimen collection, storage and shipping can be applied to future studies in low resource settings.

\section{LBP-59 Utilization Performance of Bioresources Distributed by the National Biobank of Korea}

\section{Lee, S. Park, Y. Kim, Y. Kim, J. Jeon \\ National Institute of Health, Cheongju, Korea}

National Biobank of Korea (NBK) has supported biorepositories of national cohorts and surveys including the Korea Genome and Epidemiology Study (KoGES), the Korea National Health and Nutrition Examination Survey (KNHANES). NBK has also distributed biospecimens and related genetic and epidemiological data sets to research projects approved by the distribution review committee. Here, we reports distribution statistics and bioresource utilization performance. From 2003 to 2017 , a total of 616 research projects was approved for access to NBK bioresources. The distributed bioresources were 475,675 vials of biospecimens, 614 sets of epidemiological data, and 483 sets of genetic data. Using these NBK bior- esources, 374 research papers and 28 patents were published. Regarding publication quality, the average impact factor (IF) of research papers was 5.0. The studies with both biospecimens and related data of epidemiological and genetic data produced research papers of the IF average of 8.7 whereas the studies with genetic data was 6.1 (biospecimens 5.4 / epidemiological data 5.5). Based on these results, it is necessary to collect and provide more genetic data in order to improve bioresource utilization performance. On the other hand, as NBK recently opened biofluid distribution of serum and plasma samples, it is expected that NBK bioresources will contribute to the development of diagnostic methods and new drugs.

\section{LBP-60 Enabling Detailed \& Operator-Independent Plasma QC by an Innovative Spectrophotometric Approach}

\author{
T. Martens ${ }^{1}$, K. Plasman ${ }^{1}$, T. Montoye ${ }^{1}$, E. Kwon ${ }^{2,3}$, \\ W. Ewart ${ }^{2,3}$, A. Brooks 2,3 \\ ${ }^{1}$ Unchained Labs, Gentbrugge, Belgium, ${ }^{2}$ RUCDR Infinite \\ Biologics, Rutgers University, Piscataway, New Jersey, \\ United States, ${ }^{3}$ BioProcessing Solutions Alliance, \\ Indianapolis, Indiana, United States
}

Blood plasma and serum, as a biomaterials for both molecular and functional analysis, have become increasingly important as an important analytical resource in both research and diagnostics. As these bio-molecules are present in limited amounts and are sensitive to degradation as a function of collection, processing, and storage (i.e., freeze-thaw), it is of utmost importance to qualify and quantify the quality of plasma prior to any analyses. Plasma quality is currently assessed by a rough visual inspection being imprecise, labor-intensive and operator-subjective. Moreover, many current QC tools lack the ability to predict downstream performance of the sample. This study presents the development of a novel plasma quality control approach utilizing a micro-volume UV/Vis spectrometric approach (Lunatic - Unchained Labs). This technological approach extends the capabilities of standard UV/Vis by dissecting the measured absorbance spectrum into its relevant constituents. As a result, the typical color and clarity grading are decomposed and quantified into its biologically relevant contributors: protein as major component, heme (hemolysis), bilirubin (icteric serum) and turbidity (lipidity). Using this approach, the 4 plasma QC parameters can be objectively quantified in a batch-wise method while consuming only $2 \mu \mathrm{l}$ of plasma. In this study the correlation between the visual inspection and UV/Vis approach is demonstrated, showing not only good correlation between the two methods but also the more robust, operator independent and in-depth analysis of the $\mathrm{UV} \backslash \mathrm{V}$ is method. Lastly, the study demonstrates the correlative value of relating quantitative measurements of plasma, using this new approach, with downstream analyses of both cell free nucleic acid and functional measurement quality. The implementation of this approach is the basis of a global standardization for plasma quality that can help assess the effects of pre-analytical and processing variables. 